\title{
Article \\ A Self-Adaptive-Coefficient-Double-Power Sliding Mode Control Method for Lower Limb Rehabilitation Exoskeleton Robot
}

\author{
Yuepeng Zhang ${ }^{1}$, Guangzhong Cao ${ }^{1, * \mathbb{D}}$, Wenzhou Li ${ }^{1}$, Jiangcheng Chen ${ }^{1}$, Linglong Li ${ }^{1}$ and Dongfeng Diao ${ }^{2} \mathbb{D}$ \\ 1 Guangdong Key Laboratory of Electromagnetic Control and Intelligent Robots, College of Mechatronics and \\ Control Engineering, Shenzhen University, Shenzhen 518060, China; 2050296002@email.szu.edu.cn (Y.Z.); \\ liwenzhou@email.szu.edu.cn (W.L.); jcchen@szu.edu.cn (J.C.); 2150296002@email.szu.edu.cn (L.L.) \\ 2 Institute of Nanosurface Science and Engineering (INSE), Guangdong Provincial Key Laboratory of \\ Micro/Nano Optomechatronics Engineering, Shenzhen University, Shenzhen 518060, China; \\ dfdiao@szu.edu.cn \\ * Correspondence: gzcao@szu.edu.cn; Tel.: +86-755-26534865
}

Citation: Zhang, Y.; Cao, G.; Li, W.; Chen, J.; Li, L.; Diao, D. A SelfAdaptive-Coefficient-Double-Power Sliding Mode Control Method for Lower Limb Rehabilitation Exoskeleton Robot. Appl. Sci. 2021, 11, 10329. https://doi.org/10.3390/ app112110329

Academic Editor: Giuk Lee

Received: 29 September 2021

Accepted: 1 November 2021

Published: 3 November 2021

Publisher's Note: MDPI stays neutral with regard to jurisdictional claims in published maps and institutional affiliations.

Copyright: (c) 2021 by the authors. Licensee MDPI, Basel, Switzerland. This article is an open access article distributed under the terms and conditions of the Creative Commons Attribution (CC BY) license (https:// creativecommons.org/licenses/by/ $4.0 /)$.
Abstract: Lower limb rehabilitation exoskeleton robots have the characteristics of nonlinearity and strong coupling, and they are easily disturbed during operation by environmental factors. Thus, an accurate dynamic model of the robot is difficult to obtain, and achieving trajectory tracking control of the robot is also difficult. In this article, a self-adaptive-coefficient double-power sliding mode control method is proposed to overcome the difficulty of tracking the robot trajectory. The method combines an estimated dynamic model with sliding mode control. A nonlinear control law was designed based on the robot dynamics model and computational torque method, and a compensation term of control law based on double-power reaching law was introduced to reduce the disturbance from model error and environmental factors. The self-adaptive coefficient of the compensation term of the control law was designed to adaptively adjust the compensation term to improve the anti-interference ability of the robot. The simulation and experiment results show that the proposed method effectively improves the trajectory tracking accuracy and anti-interference ability of the robot. Compared with the traditional computed torque method, the proposed method decreases the tracking error by more than $71.77 \%$. The maximum absolute error of the hip joint and knee joint remained below $0.55^{\circ}$ and $1.65^{\circ}$, respectively, in the wearable experiment of the robot.

Keywords: lower limb rehabilitation exoskeleton robot; trajectory tracking; estimated dynamic model; sliding mode control; self-adaptive-coefficient-double-power reaching law

\section{Introduction}

Stroke and spinal cord injury (SCI) are the main causes of human lower limb motor dysfunction [1-3]. Motion disability not only causes difficulties with physical control, but also serious psychological problems to the patient, which greatly reduce the quality of life of the patient. Rehabilitation training can effectively help patients with lower limb motor dysfunction improve muscle strength, restore walking ability, and reduce complications [4]. In terms of the rehabilitation process, the training mode of the robot can be divided into passive training and active training. Passive training requires the robot to drive the patient into predetermined trajectory movement, and the robot usually adopts the control method of trajectory tracking. In this article, aiming at the challenge of low tracking accuracy in trajectory tracking control, research on control methods was carried out to improve the performance of trajectory tracking control.

In the early stage of rehabilitation training, the patient's lower limbs are almost completely paralyzed (bed-ridden) and rely on external assistance to complete the training action. Traditional rehabilitation gait therapy requires multiple therapists to manually drive the patient's legs, which increases labor costs [5], or uses auxiliary equipment connected 
to the hospital bed to achieve rehabilitation training [6,7]. In the early stage of treatment, these approaches are effective, but when the patient leaves the bed for overground gait training, they cannot guarantee better results.

With the emergence of lower limb rehabilitation exoskeleton robots including LOKOMAT [8] in 2005, HAL [9] in 2008, ReWalk [10] in 2010, and ExoMotus [11] in 2018, a large number of clinical applications have shown that lower limb rehabilitation exoskeleton robots can play good auxiliary roles for patients with different degrees of injury [12,13]. Trajectory tracking control, which is one of the main control strategies in the control method of lower limb rehabilitation exoskeleton robots, is the basis and key to realizing different training modes.

The lower limb rehabilitation exoskeleton robot is a multiple-degree-of-freedom openchain mechanism composed of irregular links that have complex nonlinearity and strong coupling characteristics $[12,13]$. These robots are vulnerable to disturbances from the patient and the ground during the control process. Therefore, it is difficult to establish a complete and accurate mathematical model of the robot, which leads to difficulties in robot motion control and poor trajectory tracking accuracy. Traditional control methods including proportional-integral-derivative (PID) control [14], computed torque method [15], adaptive control [16], and robust control [17] have difficulty in meeting the actual needs of patients using lower limb rehabilitation exoskeleton robots because their trajectory tracking abilities are limited.

The sliding mode control does not depend on a mathematical model of the controlled object and has strong anti-interference ability and good robustness [18,19], consequently, sliding mode controls are widely used in robot control systems [20]. A sliding mode control divides the motion process of the robot system state into two stages. The first stage is from the initial state to the sliding surface, and the second stage gradually converges to an equilibrium point in the sliding surface [21]. However, after the system state reaches the sliding surface, it is difficult to strictly slide along the sliding surface to the equilibrium point. Instead, the system state moves back and forth across the sliding surface to approach the equilibrium point, which results in buffeting [22].

Many researchers have designed reaching laws to change the reaching speed and weaken the chattering of sliding mode controls. Islam et al. [23] and Nair et al. [24] proposed a sliding mode control based on the constant velocity reaching law for a lower limb rehabilitation exoskeleton robot. Hao et al. [25] proposed an adaptive neural network sliding mode control based on the exponential reaching law. Long et al. [26] proposed a terminal sliding mode control based on the exponential reaching law, and Chen et al. [27] proposed a terminal sliding mode control based on the exponential reaching law. Devika et al. [28] proposed a sliding mode control based on the power rate exponential reaching law by combining exponential reaching law and power reaching law. Xia et al. [29] and Kang et al. [30] proposed a terminal sliding mode control based on the double-power reaching law. The above studies revealed that the double-power reaching law had a better dynamic quality. In the double-power reaching law, the higher-order power term enables the system state to quickly reach the sliding mode surface when it is far from the surface. Additionally, the lower-order power term enables the approaching speed to decrease gradually as the system states approach the sliding mode surface, which can effectively reduce chattering. The double-power reaching law both weakens the chattering and increases the reaching speed of the system state.

In this article, a self-adaptive-coefficient double-power sliding mode control method was proposed to improve the trajectory tracking accuracy and anti-interference ability of the lower limb rehabilitation exoskeleton robot. The method combines the estimated dynamics model of the robot with sliding mode control based on the double-power reaching law to weaken chattering, and obtains an appropriate reaching speed through a designed self-adaptive-coefficient. First, based on the estimated dynamic model, the estimated control torque of the robot was obtained. Second, based on the double-power reaching law, a compensation term was designed and applied to the estimated control torque to reduce the influence of model error and environmental disturbance. Then, according to the 
Lyapunov function, a self-adaptive-coefficient of the compensation term was designed to achieve the compensation term following the model error, and a self-adaptive adjustment occurred, which improved the robust performance of the robot. Finally, taking the lower limb rehabilitation exoskeleton robot prototype in laboratory conditions as the controlled object, a co-simulation and a wearable experiment demonstrated that the self-adaptivecoefficient double-power sliding mode control method effectively improved the trajectory tracking accuracy and anti-interference ability of the robot.

\section{Structure and Dynamics Model of Lower Limb Rehabilitation Exoskeleton Robot}

\subsection{Robot Mechanical Structure}

The mechanical structure of the lower limb rehabilitation exoskeleton robot built in the laboratory is shown in Figure 1. The robot is mainly composed of the control system, torso, hip joint, thigh, knee joint, shank, ankle joint, foot, and fixative belt.

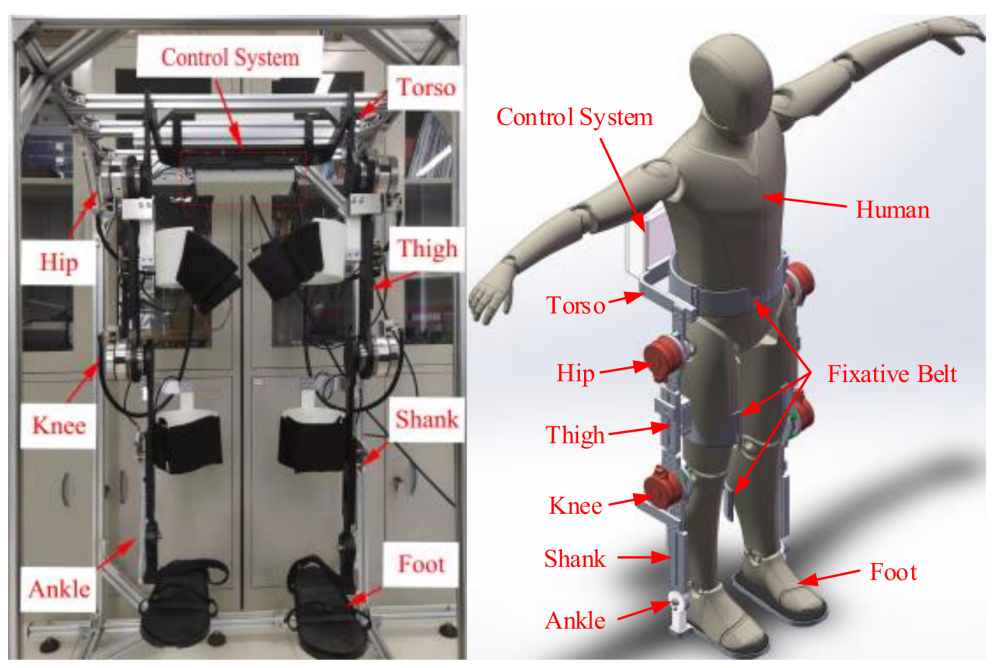

Figure 1. The prototype of the lower limb rehabilitation exoskeleton robot.

Each leg of the robot has three degrees of freedom to enable flexion or extension movements of the hip joint, knee joint, and ankle joint in the sagittal plane. The flexion or extension movements of the hip and knee joints are active movements driven by motors. In contrast, the ankle joints did not have active movement capabilities. By controlling the flexion or extension movement of the hip and knee joints of the lower limb rehabilitation exoskeleton robot, auxiliary exercises can be realized for the patient.

The parameters of each component of the lower limb rehabilitation exoskeleton robot are shown in Table 1. Among them, the torso can be adjusted by adjusting the distance between the connecting rods on both sides, and the thigh and the shank can be adjusted in length by adjusting the position of the assembly hole to adapt to the body characteristics of different patients.

Table 1. Parameters of each component of the exoskeleton robot for lower limb rehabilitation.

\begin{tabular}{ccccc}
\hline Component & Diagram & Mass/kg & Adjustable Range/mm & Size/mm \\
\hline Torso & 1.750 & High: 100 190 & 189 \\
Thigh & 3.850 & Width: 350 500 & 418 \\
Shank & 3.650 & $400 \sim 500$ & 378 \\
Foot & 1.450 & $360 \sim 500$ & High: 102 \\
& & & - & Length: 299 \\
\hline
\end{tabular}




\subsection{Robot Dynamic Model}

According to the dynamic model of the lower limb rehabilitation exoskeleton robot, the mathematical relationship between the motion of the hip, knee joints, and the control torque can be obtained. Ignoring the influence of environmental interference on the lower limb rehabilitation exoskeleton robot, a simplified structure diagram of the unilateral lower limb was established in the sagittal plane, as shown in Figure 2. The physical parameters of the simplified structure are shown in Table 2.

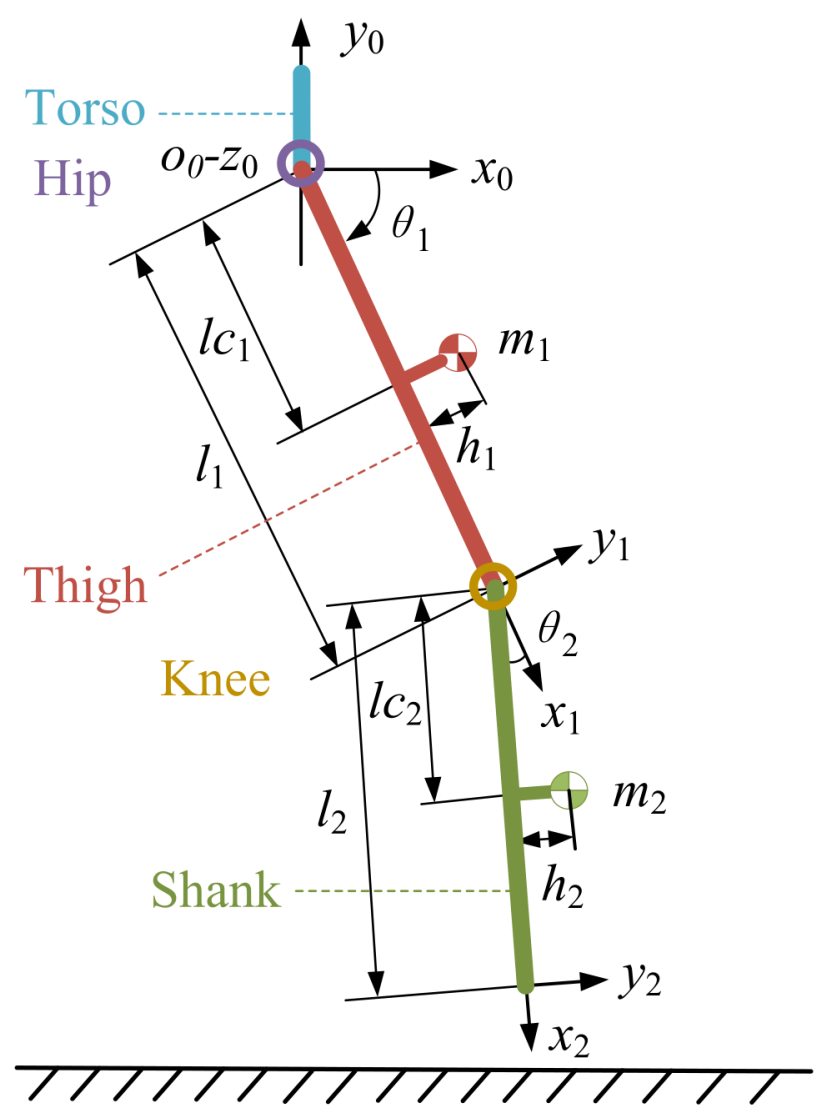

Figure 2. Simplified structural diagram of a unilateral lower limb.

Table 2. The physical parameters of the simplified structure.

\begin{tabular}{cc}
\hline Nomenclature & Description \\
\hline$\theta_{i}$ & The rotation angles of the hip and knee joints in the sagittal plane \\
$l_{i}$ & The lengths of the thigh and shank segments \\
$l_{i}$ & The distance from the driving joint of the segment to the centroid of the segment \\
$h_{i}$ & The position offset of the centroid of thigh and shank segments \\
$m_{i}$ & The masses of the thigh segment and shank segments \\
$I_{i}$ & The inertia of the thigh segment and shank segments \\
$\tau_{i}$ & The driving torque of the hip and knee joints \\
\hline
\end{tabular}

The Lagrange method is used to establish the dynamic model of the lower limb rehabilitation exoskeleton robot, which can be expressed by a second-order nonlinear differential Equation [27].

$$
M(\theta) \ddot{\theta}+C(\theta, \dot{\theta}) \dot{\theta}+G(\theta)=\tau
$$

where $\boldsymbol{\theta}=\left[\begin{array}{ll}\theta_{1} & \theta_{2}\end{array}\right]^{T}$ is the angular displacement of the hip and knee joints; $\dot{\boldsymbol{\theta}}$ is the angular velocity; $\ddot{\boldsymbol{\theta}}$ is the angular acceleration; $\boldsymbol{M}(\boldsymbol{\theta})=\left[M_{i j}\right]$ is an inertia matrix; and $\boldsymbol{C}(\boldsymbol{\theta}, \dot{\boldsymbol{\theta}})=\left[C_{i j}\right]$ 
is a centrifugal force and Coriolis force matrix, and it has a strong coupling term, $i=1 \cdots 2$, $j=1 \cdots 2 . \boldsymbol{G}(\boldsymbol{\theta})=\left[\begin{array}{ll}G_{11} & G_{21}\end{array}\right]^{\mathrm{T}}$ is a gravity term [27,31], and $\boldsymbol{\tau}=\left[\begin{array}{ll}\tau_{11} & \tau_{21}\end{array}\right]^{\mathrm{T}}$ is the control torque of the hip and knee joints. Among them,

$$
\begin{aligned}
& M_{11}=I_{1}+I_{2}+m_{1}\left(h_{1}^{2}+l c_{1}^{2}\right)+m_{2}\left(h_{2}^{2}+l_{1}^{2}+l c_{2}^{2}+2 l_{1} l c_{2} \cos \theta_{2}-2 l_{1} h_{2} \sin \theta_{2}\right) \\
& M_{12}=M_{21}=I_{2}+m_{2}\left(h_{2}^{2}+l c_{2}^{2}+l_{1} l c_{2} \cos \theta_{2}-l_{1} h_{2} \sin \theta_{2}\right) \\
& M_{22}=I_{2}+m_{2}\left(h_{2}^{2}+l c_{2}^{2}\right) \\
& C_{11}=-2 m_{2} l_{1}\left(h_{2} \cos \theta_{2}+l c_{2} \sin \theta_{2}\right)-m_{2} l_{1}\left(h_{2} \cos \theta_{2}+l c_{2} \sin \theta_{2}\right) \dot{\theta}_{1} \dot{\theta}_{2} \\
& C_{12}=C_{21}=0 \\
& C_{22}=m_{2} l_{1}\left(h_{2} \cos \theta_{2}+l c_{2} \sin \theta_{2}\right) \\
& G_{11}=m_{2} g\left(l_{2} \cos \left(\theta_{1}+\theta_{2}\right)-h_{2} \sin \left(\theta_{1}+\theta_{2}\right)+l_{1} \cos \theta_{1}\right)+m_{1} g\left(h_{1} \sin \theta_{1}+l c_{1} \cos \theta_{1}\right) \\
& G_{21}=m_{2} g\left(l c_{2} \cos \left(\theta_{1}+\theta_{2}\right)-h_{2} \sin \left(\theta_{1}+\theta_{2}\right)\right)
\end{aligned}
$$

where $l_{i}(i=1,2)$ is the length of the thigh and shank links; $l_{i}(i=1,2)$ and $h_{i}(i=1,2)$ is the centroid position of the links; $m_{i}(i=1,2)$ is the mass of the links; $I_{i}(i=1,2)$ is the inertia of the links. The parameters in Equation (2) were obtained from the 1:1 three-dimensional model constructed by the commercial software Solidworks.

For Equation (1), there are three properties of the dynamic model of the lower limb rehabilitation exoskeleton robot as follows [13,32].

Property 1. $\boldsymbol{M}(\boldsymbol{\theta})$ is a symmetric positive definite matrix and is bounded.

Property 2. The matrix $\dot{\boldsymbol{M}}(\boldsymbol{\theta})-2 \boldsymbol{C}(\boldsymbol{\theta}, \dot{\boldsymbol{\theta}})$ is skew-symmetry [13,32,33], and satisfies the following relationship:

$$
\xi^{\mathrm{T}}(\dot{M}(\boldsymbol{\theta})-2 C(\boldsymbol{\theta}, \dot{\boldsymbol{\theta}})) \xi=0, \forall \xi \in \mathrm{R}^{2 \times 2}
$$

Property 3. A constant parameter vector describes the quality characteristics of the robot $\boldsymbol{P} \in \mathbf{R}^{l}$ [34] and a known regression matrix about the function of robot joint variables $\boldsymbol{Y}(\boldsymbol{\theta}, \dot{\boldsymbol{\theta}}, \boldsymbol{\rho}, \boldsymbol{\vartheta}) \in \mathbf{R}^{2 \times l}$, making $\boldsymbol{M}(\boldsymbol{\theta}), \boldsymbol{C}(\boldsymbol{\theta}, \dot{\boldsymbol{\theta}})$, and $\boldsymbol{G}(\boldsymbol{\theta})$ satisfy the following relationship.

$$
M(\theta) \vartheta+C(\theta, \dot{\theta}) \rho+G(\theta)=Y(\theta, \dot{\theta}, \rho, \vartheta) P
$$

\section{Self-Adaptive-Coefficient Double-Power Sliding Mode Control Algorithm}

The control block diagram of the self-adaptive-coefficient double-power sliding mode control method is shown in Figure 3. The design is mainly divided into three parts. Part (I): the dynamic model of the lower limb rehabilitation exoskeleton robot shown in Equation (1) is regarded as an estimated dynamic model. The estimated control torque $\bar{u}$ is obtained to reduce the nonlinear and strong coupling influences on the robot. Part (II): based on a double-power reaching law, we designed a compensation term $\tau_{c}$, the sum of the compensation term $\tau_{c}$, and the estimated control torque $\bar{u}$ is used as the actual control torque $\tau$ of the robot to reduce the effects of model error and environmental interference. Part (III): a self-adaptive coefficient $\Lambda$ was designed for the compensation term $\emptyset_{c}$, and the self-adaptive-coefficient double-power compensation term (SACDPCT) $\Lambda \tau_{c}$ adjusts adaptively, improving the robust performance of the robot trajectory tracking control. 


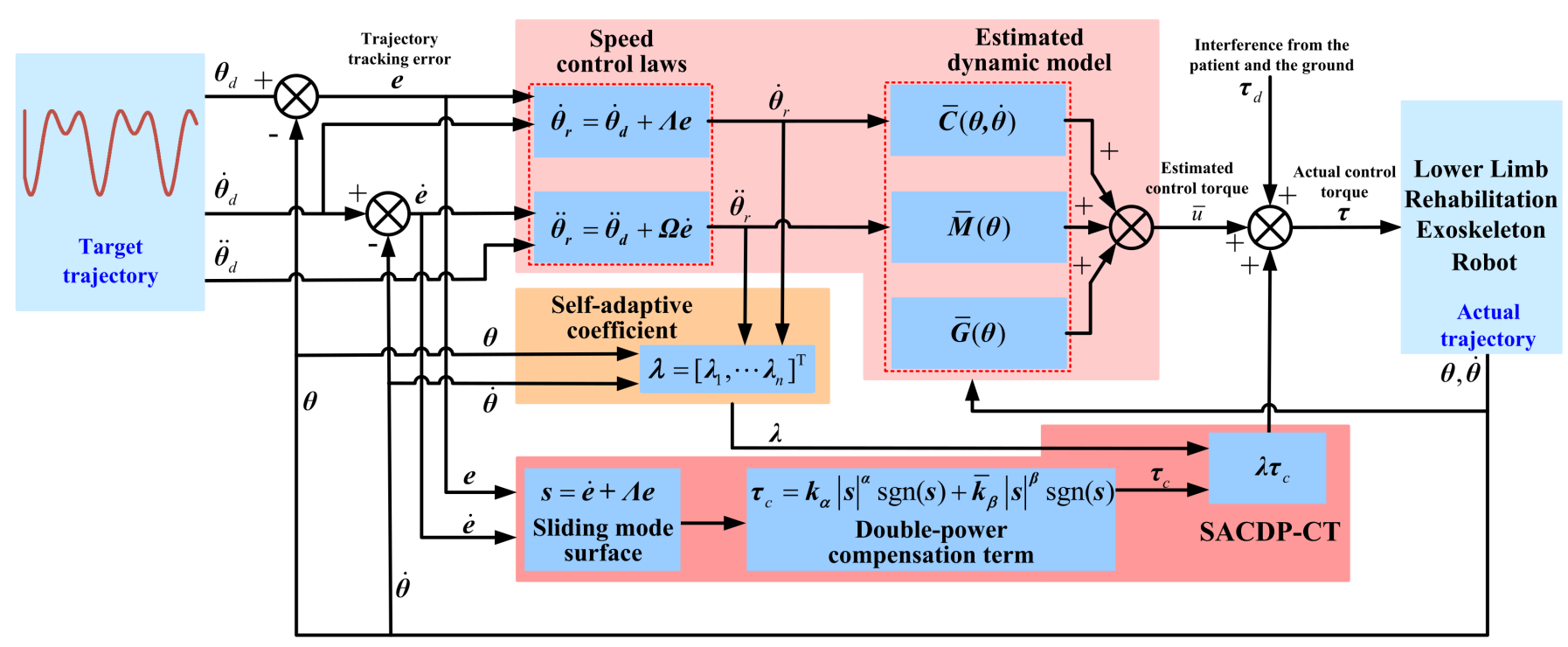

Figure 3. The control block diagram.

\subsection{Design of Control Law Based on Estimated Model}

The patient and the ground environment inevitably interfere with the lower limb rehabilitation exoskeleton robot, which leads to the model error between the established dynamic model and the actual dynamic model. Therefore, this paper regards the established dynamic model as an estimated dynamic model. Using the computed torque method, we designed an estimated control torque based on the estimated dynamic model.

The established dynamic model of the lower limb rehabilitation exoskeleton robot in this paper is shown in Equation (1). Taking the established dynamic model as an estimated dynamic model, we rewrite Equation (1) as follows.

$$
\bar{M}(\theta) \ddot{\theta}+\bar{C}(\theta, \dot{\theta}) \dot{\theta}+\bar{G}(\theta)=\bar{\tau}
$$

where $\overline{\boldsymbol{M}}(\boldsymbol{\theta})$ is an estimate of the actual inertia matrix $\boldsymbol{M}(\boldsymbol{\theta}) ; \overline{\boldsymbol{C}}(\boldsymbol{\theta}, \dot{\boldsymbol{\theta}}) \dot{\boldsymbol{\theta}}$ is an estimate of the actual centrifugal force and Coriolis force matrix $\boldsymbol{C}(\boldsymbol{\theta}, \dot{\boldsymbol{\theta}}) ; \overline{\boldsymbol{G}}(\boldsymbol{\theta})$ is an estimate of the actual gravity term $G(\theta)$; and $\bar{\tau}$ is an estimate of the actual control torque $\tau$. Therefore, it can be seen from Equation (6):

$$
\left[\begin{array}{ll}
\bar{M}_{11} & \bar{M}_{12} \\
\bar{M}_{21} & \bar{M}_{22}
\end{array}\right] \ddot{\boldsymbol{\theta}}+\left[\begin{array}{ll}
\bar{C}_{11} & \bar{C}_{12} \\
\bar{C}_{21} & \bar{C}_{22}
\end{array}\right] \dot{\boldsymbol{\theta}}+\left[\begin{array}{l}
\bar{G}_{11} \\
\bar{G}_{21}
\end{array}\right]=\left[\begin{array}{l}
\bar{\emptyset}_{11} \\
\bar{\emptyset}_{21}
\end{array}\right]
$$

Considering that the patient and the ground environment will inevitably interfere with the lower limb rehabilitation exoskeleton robot, the actual dynamic model of the lower limb rehabilitation exoskeleton robot can be expressed as follows.

$$
\boldsymbol{M}(\boldsymbol{\theta}) \ddot{\boldsymbol{\theta}}+\boldsymbol{C}(\boldsymbol{\theta}, \dot{\boldsymbol{\theta}}) \dot{\boldsymbol{\theta}}+G(\boldsymbol{\theta})+\tau_{d}=\tau
$$

where $\tau_{d}=\left[\begin{array}{ll}\emptyset_{d 11} & \emptyset_{d 21}\end{array}\right]^{\mathrm{T}}$ is interference, which reflects the interference from the patient and the ground. $\left|\tau_{d}\right| \leq f$, and $f$ is a positive constant matrix.

Because the actual inertial parameters of the lower limb rehabilitation exoskeleton robot cannot be accurately obtained. The actual inertia matrix $\boldsymbol{M}(\boldsymbol{\theta})$, the actual centrifugal force, and Coriolis force matrix $C(\boldsymbol{\theta}, \dot{\boldsymbol{\theta}})$, the actual gravity term $\boldsymbol{G}(\boldsymbol{\theta})$, and the actual interference $\tau_{d}$ are unknown. Based on the computed torque method, a nonlinear feedback control law was designed as the estimated control torque $\bar{u}$ for each joint of the robot and expressed as follows.

$$
\overline{\boldsymbol{u}}=\overline{\boldsymbol{M}}(\boldsymbol{\theta}) \ddot{\boldsymbol{\theta}}_{r}+\overline{\boldsymbol{C}}(\boldsymbol{\theta}, \dot{\boldsymbol{\theta}}) \dot{\boldsymbol{\theta}}_{r}+\overline{\boldsymbol{G}}(\boldsymbol{\theta})
$$


where $\dot{\boldsymbol{\theta}}_{r}$ and $\ddot{\boldsymbol{\theta}}_{r}$ are the speed control laws to be designed. During the trajectory tracking control of the lower limb rehabilitation exoskeleton robot, the robot needs to track a timevarying target trajectory $\boldsymbol{\theta}_{\boldsymbol{d}}(t)$. To perform the trajectory tracking of the robot, the speed control laws $\dot{\boldsymbol{\theta}}_{r}$ and $\ddot{\boldsymbol{\theta}}_{r}$ were designed as follows

$$
\left\{\begin{array}{l}
\dot{\theta}_{r}=\dot{\theta}_{d}+\Lambda e \\
\ddot{\theta}_{r}=\ddot{\theta}_{d}+\Omega \dot{e}
\end{array}\right.
$$

where $\dot{\boldsymbol{\theta}}_{d}$ is the target angular velocity; $\ddot{\boldsymbol{\theta}}_{d}$ is the target angular acceleration; $\boldsymbol{\Lambda}$ and $\boldsymbol{\Omega}$ are the positive diagonal matrix; $\boldsymbol{\Lambda}=\operatorname{diag}\left(\Lambda_{1}, \cdots, \Lambda_{n}\right) ; \boldsymbol{\Omega}=\operatorname{diag}\left(\Omega_{1}, \cdots, \Omega_{n}\right) ; \boldsymbol{e}$ is the trajectory tracking error; and $\boldsymbol{e}=\boldsymbol{\theta}_{\boldsymbol{d}}-\boldsymbol{\theta}$.

According to Equations (6), (8) and (9), based on the computed torque method and the estimated dynamic model, the estimated control torque for each joint of the lower limb rehabilitation exoskeleton robot was designed as follows:

$$
\overline{\boldsymbol{u}}=\left[\begin{array}{ll}
\bar{M}_{11} & \bar{M}_{12} \\
\bar{M}_{21} & \bar{M}_{22}
\end{array}\right]\left(\ddot{\boldsymbol{\theta}}_{\boldsymbol{d}}+\boldsymbol{\Omega} \dot{\boldsymbol{e}}\right)+\left[\begin{array}{ll}
\overline{\mathrm{C}}_{11} & \overline{\mathrm{C}}_{12} \\
\overline{\mathrm{C}}_{21} & \overline{\mathrm{C}}_{22}
\end{array}\right]\left(\dot{\boldsymbol{\theta}}_{\boldsymbol{d}}+\boldsymbol{\Lambda} \boldsymbol{e}\right)+\left[\begin{array}{l}
\bar{G}_{11} \\
\bar{G}_{21}
\end{array}\right]
$$

\subsection{Design of Compensation Item Based on Double-Power Reaching Law}

A sliding mode control allows a robot to maintain system stability under the conditions in which parameters change and environmental interference occurs through the switching of the self-adaptive control [35]. To reduce the influence of model error and environmental interference on the trajectory tracking of the lower limb rehabilitation exoskeleton robot, its trajectory tracking was achieved by combining the estimated control torque with the sliding mode control method based on a double-power reaching law.

The sliding mode surface $s \in \mathbf{R}^{n}$ for the sliding mode control was designed as follows:

$$
s=\dot{\theta}_{r}-\dot{\theta}=\dot{e}+\Lambda e
$$

where the interference $\tau_{d}$ is bounded. Based on the double-power reaching law, the compensation term $\tau_{c}$ for the actual control torque was designed as follows:

$$
\tau_{c}=k_{\alpha}|s|^{\alpha} \operatorname{sgn}(s)+\bar{k}_{\beta}|s|^{\beta} \operatorname{sgn}(s)
$$

where $\bar{k}_{\beta}=k_{\beta}+\frac{f}{|s|^{\beta}} ; \operatorname{sgn}(s)$ is the symbolic function; and $k_{\alpha} \geq 1, k_{\beta} \geq 1, \alpha>1$, $0<\beta<1$.

Because $\alpha>1$ and $0<\beta<1$, during the entire sliding phase $(s \rightarrow 0)$, when the system state of the lower limb rehabilitation exoskeleton robot is far from the equilibrium point (e.g., $s>1$ ), the compensation term $\tau_{c}$ is determined by the higher-order power term $k_{\alpha}|s|^{\alpha} \operatorname{sgn}(s)$. At this time, $\alpha>1$ makes the high-order power term larger, which effectively increases the approach speed. However, when the system state of the robot is close to the sliding mode surface (e.g., $s<1$ ), the compensation term $\tau_{c}$ is determined by the low-order power term $\boldsymbol{k}_{\boldsymbol{\beta}}|\boldsymbol{s}|^{\beta} \operatorname{sgn}(s)$. At this point, $0<\boldsymbol{\beta}<1$ makes the low-order power term smaller and reduces the reaching speed, which effectively avoids an excessive reaching speed and reduces chattering of the system state.

\subsection{Design of Self-Adaptive-Coefficient-Double-Power Sliding Mode Control Method}

According to Equations (10) and (12), combining the estimated dynamic model of the lower limb rehabilitation exoskeleton robot and the compensation term based on the 
double-power reaching law, the control law of the double-power sliding mode control method can be expressed as follows.

$$
\begin{aligned}
\boldsymbol{\tau} & =\boldsymbol{u}=\overline{\boldsymbol{u}}+\boldsymbol{\tau}_{c}=\overline{\boldsymbol{M}}(\boldsymbol{\theta}) \ddot{\boldsymbol{\theta}}_{r}+\overline{\boldsymbol{C}}(\boldsymbol{\theta}, \dot{\boldsymbol{\theta}}) \dot{\boldsymbol{\theta}}_{r}+\overline{\boldsymbol{G}}(\boldsymbol{\theta})+\boldsymbol{\tau}_{c} \\
& =\left[\begin{array}{ll}
\bar{M}_{11} & \bar{M}_{12} \\
\bar{M}_{21} & \bar{M}_{22}
\end{array}\right] \ddot{\boldsymbol{\theta}}_{r}+\left[\begin{array}{ll}
\bar{C}_{11} & \bar{C}_{12} \\
\bar{C}_{21} & \bar{C}_{22}
\end{array}\right] \ddot{\boldsymbol{\theta}}_{r}+\left[\begin{array}{c}
\bar{G}_{11} \\
\bar{G}_{21}
\end{array}\right]+\boldsymbol{k}_{\alpha}|\boldsymbol{s}|^{\alpha} \operatorname{sgn}(\boldsymbol{s})+\overline{\boldsymbol{k}}_{\boldsymbol{\beta}}|\boldsymbol{s}|^{\beta} \operatorname{sgn}(\boldsymbol{s})
\end{aligned}
$$

We defined the Lyapunov function as follows.

$$
\boldsymbol{V}(t)=0.5 \boldsymbol{s}^{\mathrm{T}} \boldsymbol{M}(\boldsymbol{\theta}) \boldsymbol{s}
$$

According to Equations (3), (7) and (13), the derivative of Equation (14) with respect to time $t$ is expressed as follows:

$$
\begin{aligned}
\dot{\boldsymbol{V}}(t) & =\boldsymbol{s}^{\mathrm{T}} \boldsymbol{M}(\boldsymbol{\theta}) \dot{\boldsymbol{s}}+\frac{1}{2} \boldsymbol{s}^{\mathrm{T}}(2 \boldsymbol{C}(\boldsymbol{\theta}, \dot{\boldsymbol{\theta}})) \boldsymbol{s}=\boldsymbol{s}^{\mathrm{T}}\left[\boldsymbol{M}(\boldsymbol{\theta})\left(\ddot{\boldsymbol{\theta}}_{r}-\ddot{\boldsymbol{\theta}}\right)+\boldsymbol{C}(\boldsymbol{\theta}, \dot{\boldsymbol{\theta}})\left(\dot{\boldsymbol{\theta}}_{r}-\dot{\boldsymbol{\theta}}\right)\right] \\
& =\boldsymbol{s}^{\mathrm{T}}\left[\boldsymbol{M}(\boldsymbol{\theta}) \ddot{\boldsymbol{\theta}}_{r}+\boldsymbol{C}(\boldsymbol{\theta}, \dot{\boldsymbol{\theta}}) \dot{\boldsymbol{\theta}}_{r}+\boldsymbol{G}(\boldsymbol{\theta})+\boldsymbol{\tau}_{d}-\boldsymbol{u}\right] \\
& =\boldsymbol{s}^{\mathrm{T}}\left[(\boldsymbol{M}(\boldsymbol{\theta})-\overline{\boldsymbol{M}}(\boldsymbol{\theta})) \ddot{\boldsymbol{\theta}}_{r}+(\boldsymbol{C}(\boldsymbol{\theta}, \dot{\boldsymbol{\theta}})-\bar{C}(\boldsymbol{\theta}, \dot{\boldsymbol{\theta}})) \dot{\boldsymbol{\theta}}_{r}+(\boldsymbol{G}(\boldsymbol{\theta})-\overline{\boldsymbol{G}}(\boldsymbol{\theta}))+\boldsymbol{\tau}_{d}-\boldsymbol{\tau}_{c}\right] \\
& =\boldsymbol{s}^{\mathrm{T}}\left[\tilde{\boldsymbol{M}}(\boldsymbol{\theta}) \ddot{\boldsymbol{\theta}}_{r}+\tilde{\boldsymbol{C}}(\boldsymbol{\theta}, \dot{\boldsymbol{\theta}}) \dot{\boldsymbol{\theta}}_{r}+\tilde{\boldsymbol{G}}(\boldsymbol{\theta})+\boldsymbol{\tau}_{d}-\boldsymbol{\tau}_{c}\right]
\end{aligned}
$$

where $\tilde{M}(\boldsymbol{\theta})$ is the inertial matrix error and $\tilde{\boldsymbol{M}}(\boldsymbol{\theta})=\boldsymbol{M}(\boldsymbol{\theta})-\overline{\boldsymbol{M}}(\boldsymbol{\theta}) . \tilde{\boldsymbol{C}}(\boldsymbol{\theta}, \dot{\boldsymbol{\theta}})$ is the centrifugal and Coriolis forces error, and $\tilde{C}(\boldsymbol{\theta}, \dot{\boldsymbol{\theta}})=\boldsymbol{C}(\boldsymbol{\theta}, \dot{\boldsymbol{\theta}})-\bar{C}(\boldsymbol{\theta}, \dot{\boldsymbol{\theta}})$. Here, $\tilde{\boldsymbol{G}}(\boldsymbol{\theta})$ is the gravity term error, where $\tilde{G}(\theta)=G(\theta)-\bar{G}(\theta)$.

Equations (4) and (15) can be expressed as follows.

$$
\begin{aligned}
\dot{\boldsymbol{V}}(t) & =\boldsymbol{s}^{\mathrm{T}}\left[\tilde{\boldsymbol{M}}(\boldsymbol{\theta}) \ddot{\boldsymbol{\theta}}_{r}+\tilde{\boldsymbol{C}}(\boldsymbol{\theta}, \dot{\boldsymbol{\theta}}) \dot{\boldsymbol{\theta}}_{r}+\tilde{\boldsymbol{G}}(\boldsymbol{\theta})+\boldsymbol{\tau}_{d}-\boldsymbol{\tau}_{c}\right] \\
& =\boldsymbol{s}^{\mathrm{T}}\left[\boldsymbol{Y}\left(\boldsymbol{\theta}, \dot{\boldsymbol{\theta}}, \dot{\boldsymbol{\theta}}_{r}, \ddot{\boldsymbol{\theta}}_{r}\right) \tilde{\boldsymbol{p}}+\boldsymbol{\tau}_{d}-\boldsymbol{\tau}_{c}\right]
\end{aligned}
$$

where $\boldsymbol{Y}\left(\boldsymbol{\theta}, \dot{\boldsymbol{\theta}}, \dot{\boldsymbol{\theta}}_{r}, \ddot{\boldsymbol{\theta}}_{r}\right) \tilde{\boldsymbol{p}}$ is the linear expression of the model error; $\boldsymbol{Y}\left(\boldsymbol{\theta}, \dot{\boldsymbol{\theta}}, \dot{\boldsymbol{\theta}}_{r}, \ddot{\boldsymbol{\theta}}_{r}\right)$ is a known regression matrix about the function of robot joint variables; $\boldsymbol{Y}\left(\boldsymbol{\theta}, \dot{\boldsymbol{\theta}}, \dot{\boldsymbol{\theta}}_{r}, \ddot{\boldsymbol{\theta}}_{r}\right)=\left[\boldsymbol{Y}_{i j}^{r}\right] ;\left|Y_{i j}^{r}\right| \leq \bar{Y}_{i j}^{r}$; and $\tilde{p}$ is a constant parameter vector that describes the quality characteristics of the robot, where $\tilde{\boldsymbol{p}}=\left[\widetilde{p}_{1}, \cdots, \widetilde{p}_{j}\right]^{\mathrm{T}},\left|\widetilde{p}_{j}\right| \leq \varepsilon_{j}, i=1,2$, and $j=1, \cdots, l$. Therefore,

$$
\begin{aligned}
Y\left(\theta, \dot{\theta}, \dot{\theta}_{r}, \ddot{\theta}_{r}\right) \tilde{p} & =\left[Y_{i j}^{r}\right] \cdot\left[\tilde{p}_{1}, \cdots, \tilde{p}_{j}\right]^{\mathrm{T}} \\
& =\left[\begin{array}{ccc}
Y_{11}^{r} & \cdots & Y_{1 l}^{r} \\
\vdots & Y_{i j}^{r} & \vdots \\
Y_{n 1}^{r} & \cdots & Y_{n l}^{r}
\end{array}\right] \cdot\left[\begin{array}{c}
\widetilde{p}_{1} \\
\vdots \\
\widetilde{p}_{l}
\end{array}\right] \leq\left[\begin{array}{ccc}
\bar{Y}_{11}^{r} & \cdots & \bar{Y}_{1 l}^{r} \\
\vdots & Y_{i j}^{r} & \vdots \\
\bar{Y}_{n 1}^{r} & \cdots & \bar{Y}_{n l}^{r}
\end{array}\right] \cdot\left[\begin{array}{c}
\varepsilon_{1} \\
\vdots \\
\varepsilon_{l}
\end{array}\right]=Y_{i j}^{r} \cdot \varepsilon_{j}
\end{aligned}
$$

From Equation (16), $\boldsymbol{Y}\left(\boldsymbol{\theta}, \dot{\boldsymbol{\theta}}, \dot{\boldsymbol{\theta}}_{r}, \ddot{\boldsymbol{\theta}}_{r}\right) \tilde{\boldsymbol{p}}, \boldsymbol{\tau}_{c}$, and $\boldsymbol{\tau}_{d}$ determine whether the control system of the lower limb rehabilitation exoskeleton robot is closed-loop stable. To ensure the stable convergence of the control system, it is necessary to be satisfied as follows.

$$
\dot{\boldsymbol{V}}(t)=\boldsymbol{s}^{\mathrm{T}}\left[\boldsymbol{Y}\left(\boldsymbol{\theta}, \dot{\boldsymbol{\theta}}, \dot{\boldsymbol{\theta}}_{r}, \ddot{\boldsymbol{\theta}}_{r}\right) \widetilde{\boldsymbol{p}}-\left(\boldsymbol{\tau}_{c}-\boldsymbol{\tau}_{\mathrm{d}}\right)\right] \leq 0
$$

To enhance the stable convergence of the control system and ensure that the compensation term follows the model error, adjusts adaptively, and improves the anti-interference 
ability of the robot control system, a self-adaptive-coefficient $\Lambda$ was designed for the compensation term $\tau_{c}$ and $\Lambda$ as follows.

$$
\boldsymbol{\Lambda}=\left[\boldsymbol{\Lambda}_{1}, \cdots \boldsymbol{\Lambda}_{n}\right]^{\mathrm{T}}, \Lambda_{i}=\left\{\begin{array}{l}
\sum_{j=1}^{l} \bar{Y}_{i j}^{r} \varepsilon_{j}, \boldsymbol{\Lambda}_{i} \geq 1 \\
1, \boldsymbol{\Lambda}_{i}<1
\end{array}\right.
$$

According to Equations (12) and (19), we rewrite Equation (16) as follows.

$$
\begin{aligned}
\dot{\boldsymbol{V}}(t) & =\boldsymbol{s}^{\mathrm{T}}\left[\boldsymbol{Y}\left(\theta, \dot{\boldsymbol{\theta}}, \dot{\boldsymbol{\theta}}_{r}, \ddot{\boldsymbol{\theta}}_{r}\right) \tilde{\boldsymbol{p}}-\left(\boldsymbol{\Lambda} \boldsymbol{\tau}_{c}-\boldsymbol{\tau}_{d}\right)\right] \\
& =\sum_{i=1}^{n} \sum_{j=1}^{l} s_{i} Y_{i j}^{r} \widetilde{p}_{j}-\sum_{i=1}^{n} s_{i}\left[\boldsymbol{\Lambda}_{i} k_{\boldsymbol{\alpha} i}\left|s_{i}\right|^{\alpha} \operatorname{sgn}\left(s_{i}\right)-\boldsymbol{\Lambda}_{i} k_{\boldsymbol{\beta} i}\left|s_{i}\right|^{\boldsymbol{\beta}} \operatorname{sgn}\left(s_{i}\right)-\left(\boldsymbol{\Lambda}_{i} f_{i} \operatorname{sgn}\left(s_{i}\right)-\boldsymbol{\tau}_{d}\right)\right] \\
& =\sum_{i=1}^{n} \sum_{j=1}^{l} Y_{i j}^{r} \widetilde{p}_{j} s_{i}-k_{\alpha i} \sum_{i=1}^{n} \sum_{j=1}^{l} \bar{Y}_{i j}^{r} \varepsilon_{j}\left|s_{i}\right|^{\alpha+1}-k_{\beta i} \sum_{i=1}^{n} \sum_{j=1}^{l} \bar{Y}_{i j}^{r} \varepsilon_{j}\left|s_{i}\right|^{\boldsymbol{\beta}+1}-\sum_{i=1}^{n}\left(\boldsymbol{\Lambda}_{i} f_{i}\left|s_{i}\right|-\boldsymbol{\tau}_{d i} s_{i}\right) \\
& \leq-k_{\boldsymbol{\beta} i} \sum_{i=1}^{n} \sum_{j=1}^{l} \bar{Y}_{i j}^{r} \varepsilon_{j}\left|s_{i}\right|^{\boldsymbol{\beta}+1}=-k_{\boldsymbol{\beta} i} \sum_{i=1}^{n} \boldsymbol{\Lambda}_{i}\left|s_{i}\right|^{\boldsymbol{\beta}+1} \leq-\sum_{i=1}^{n} \boldsymbol{\Lambda}_{i}\left|s_{i}\right|^{\boldsymbol{\beta}+1} \leq 0
\end{aligned}
$$

From Equation (20), we can see that $\dot{V}(t) \leq 0$. According to LaSalle's theorem [36], when $\dot{V} \equiv 0, s \equiv 0$. Thus $\dot{e} \equiv 0, e \equiv 0$. Therefore, the introduction of the self-adaptivecoefficient $\boldsymbol{\Lambda}$ can make $\dot{\boldsymbol{V}}(t) \leq 0$ and ensure that the control system of the lower limb rehabilitation exoskeleton robot is closed-loop stable.

In addition, the self-adaptive-coefficient $\boldsymbol{\Lambda}$ is determined by the model error $\boldsymbol{\gamma}\left(\boldsymbol{\theta}, \dot{\boldsymbol{\theta}}_{,}, \dot{\boldsymbol{\theta}}_{r}, \ddot{\boldsymbol{\theta}}_{r}\right) \tilde{\boldsymbol{p}}$. Therefore, $\boldsymbol{\Lambda} \tau_{c}$ can follow the model error and self-adaptive adjustment, thereby enhancing the anti-interference ability of the control system.

In summary, the control law of the self-adaptive-coefficient double-power sliding mode control method proposed in this paper is shown in Equation (21) where $u$ is the actual control torque for each joint of a lower limb rehabilitation exoskeleton robot.

$$
\begin{aligned}
\boldsymbol{\tau} & =\boldsymbol{u}=\overline{\boldsymbol{u}}+\boldsymbol{\Lambda} \boldsymbol{\tau}_{c}=\overline{\boldsymbol{M}}(\boldsymbol{\theta}) \ddot{\boldsymbol{\theta}}_{r}+\overline{\boldsymbol{C}}(\boldsymbol{\theta}, \dot{\boldsymbol{\theta}}) \dot{\boldsymbol{\theta}}_{r}+\overline{\boldsymbol{G}}(\boldsymbol{\theta})+\boldsymbol{\Lambda} \boldsymbol{\tau}_{c} \\
& =\left[\begin{array}{ll}
\bar{M}_{11} & \bar{M}_{12} \\
\bar{M}_{21} & \bar{M}_{22}
\end{array}\right] \ddot{\boldsymbol{\theta}}_{r}+\left[\begin{array}{ll}
\bar{C}_{11} & \bar{C}_{12} \\
\bar{C}_{21} & \bar{C}_{22}
\end{array}\right] \dot{\boldsymbol{\theta}}_{r}+\left[\begin{array}{l}
\bar{G}_{11} \\
\bar{G}_{21}
\end{array}\right]+ \\
& {\left[\begin{array}{c}
\Lambda_{1}\left(k_{f f 1}\left|\boldsymbol{s}_{1}\right| f^{f f} \operatorname{sgn}\left(\boldsymbol{s}_{1}\right)+\bar{k}_{\beta 1}\left|\boldsymbol{s}_{1}\right|^{\beta} \operatorname{sgn}\left(\boldsymbol{s}_{1}\right)\right) \\
\Lambda_{2}\left(k_{f f 2}\left|\boldsymbol{s}_{2}\right|{ }^{f f} \operatorname{sgn}\left(\boldsymbol{s}_{2}\right)+\bar{k}_{\beta 2}\left|\boldsymbol{s}_{2}\right|^{\beta} \operatorname{sgn}\left(\boldsymbol{s}_{2}\right)\right)
\end{array}\right] }
\end{aligned}
$$

\section{Simulation and Experimental Analysis}

To verify the effectiveness of the self-adaptive-coefficient double-power sliding mode control method proposed in this paper, we conducted a simulation and an experimental analysis. First, a joint simulation model of the lower limb rehabilitation exoskeleton robot and the patient was established using MATLAB/Simulink and RecurDyn dynamics software. Then, the simulation analysis of the robot trajectory tracking control was carried out under interference from the patient. We compared the control accuracy and antiinterference ability of the three control methods (i.e., the self-adaptive-coefficient doublepower sliding mode control method, the double-power sliding mode control method, and the traditional computed torque method). Finally, an experiment was conducted on the lower limb rehabilitation exoskeleton robot prototype with selected healthy testers.

\subsection{Simulation Model}

An adult male with a height of $1755 \mathrm{~mm}$ and a weight of $70 \mathrm{~kg}$ was selected as a representative patient for the lower limb rehabilitation exoskeleton robot. The patient and the robot were rigidly connected, and their movements were identical. As shown in 
Figure 4, we utilized MATLAB/Simulink and RecurDyn dynamics software to build a human-machine coupling system simulation model.

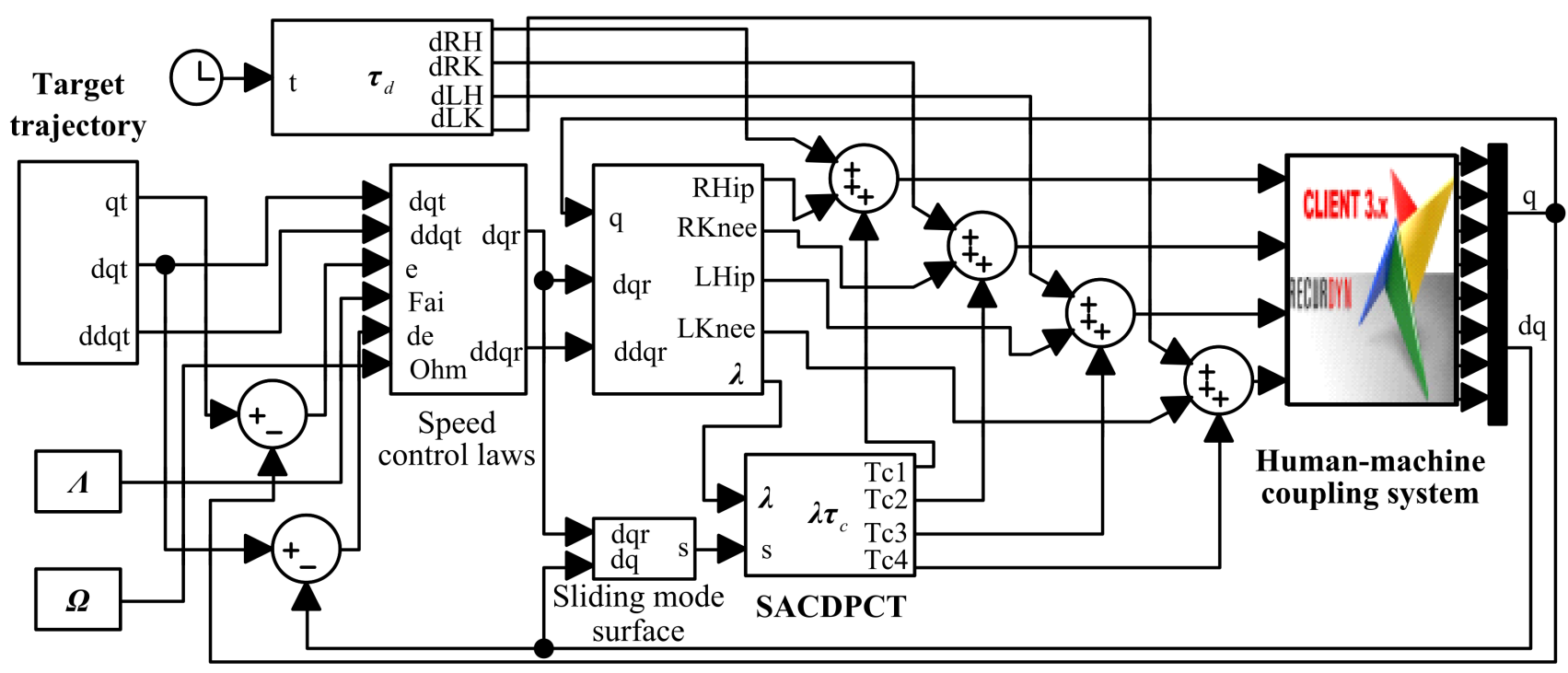

Figure 4. The control simulation in MATLAB/Simulink.

The human-machine coupling system has five segments: the torso, the right thigh, the left thigh, the right shank and foot, and the left shank and foot. The parameters of each body segment are shown in Table 3. Among these, the parameters of the right thigh and the left thigh were the same, and the parameters of the right shank and foot and left shank and foot were the same.

Table 3. The parameters of each body segment.

\begin{tabular}{|c|c|c|c|c|c|}
\hline \multirow{2}{*}{ Segments } & \multirow{2}{*}{$\begin{array}{l}\text { Mass } \\
m_{i} / \mathrm{kg}\end{array}$} & \multirow{2}{*}{$\begin{array}{c}\text { Length } \\
l_{i} / \mathrm{mm}\end{array}$} & \multicolumn{2}{|c|}{ Centroid Position } & \multirow{2}{*}{$\begin{array}{c}\text { Inertia } \\
I_{i} /\left(\mathrm{kg} \cdot \mathrm{m}^{2}\right)\end{array}$} \\
\hline & & & $l c_{i} / \mathrm{mm}$ & $h_{i} / \mathrm{mm}$ & \\
\hline Torso & 48.21 & 874.0 & 358.8 & 16.3 & 2.4093 \\
\hline Thigh & 14.05 & 418.0 & 178.5 & 14.6 & 0.2440 \\
\hline Shank \& Foot & 6.72 & 481.0 & 281.8 & 17.8 & 0.2142 \\
\hline
\end{tabular}

\subsection{Simulation Parameters}

The simulation time was $5 \mathrm{~s}$, the simulation step length was $0.001 \mathrm{~s}$, and the gait period was $2 \mathrm{~s}$. The walking gait data of a normal person with a walking speed of $1.2 \mathrm{~m} / \mathrm{s}$ was selected as the expected trajectory of each joint. The fitting curve is as follows.

$$
\left\{\begin{array}{l}
\theta_{R H}=7.52-12.94 \sin (2 \pi t-0.29)+4.78 \sin (4 \pi t-0.64) \\
\theta_{R K}=-22.7+23.69 \sin (2 \pi t+1.02)-18.54 \sin (4 \pi t-0.41) \\
\theta_{L H}=7.52+12.94 \sin (2 \pi t-0.29)+4.78 \sin (4 \pi t-0.64) \\
\theta_{L K}=-22.7-23.69 \sin (2 \pi t+1.02)-18.54 \sin (4 \pi t-0.41) .
\end{array}\right.
$$

where $\theta_{R H}$ is the desired trajectory of the right hip joint; $\theta_{R K}$ is the desired trajectory of the right knee joint; $\theta_{L H}$ is the desired trajectory of the left hip joint; and $\theta_{L K}$ is the desired trajectory of the left knee joint.

From Equation (21), the self-adaptive-coefficient double-power sliding mode control method proposed in this paper was recorded as SACDP-SMC. We set $\bar{Y}_{i j}^{r}=\left|Y_{i j}^{r}\right|+0.1$, $\varepsilon_{j}=\left|\widetilde{p}_{j}\right|+0.5, \widetilde{p}_{j}=0.05 p_{j}, \bar{p}_{j}=0.95 p_{j} f=\left|\tau_{d}\right|+0.5$, and the control parameter as the optimal parameter obtained after 120 online optimizations. $\boldsymbol{\Lambda}=\operatorname{diag}[100,100,100,100]$, 
$\boldsymbol{\Omega}=\operatorname{diag}[15,15,15,15], \boldsymbol{k}_{\boldsymbol{\alpha}}=[6,6.5,6,6.5]^{\mathrm{T}}, \boldsymbol{\alpha}=[5 / 3,5 / 3,5 / 3,5 / 3]^{\mathrm{T}}, \boldsymbol{k}_{\beta}=[1,1.5,1,1.5]^{\mathrm{T}}$, and $\beta=[3 / 5,3 / 5,3 / 5,3 / 5]^{\mathrm{T}}$.

To demonstrate the superiority of SACDP-SMC, we named it Method 1 and compared it with the following two control methods:

Method 2: To highlight the effect of the self-adaptive-coefficient $\boldsymbol{\Lambda}$, the double-power sliding mode control method was adopted and recorded as DP-SMC. The actual control torque of each joint of the robot is shown in Equation (13); the control parameters were the same as those described for SACDP-SMC.

Method 3: To highlight the compensation effect of the double-power sliding mode control, a traditional computed torque method based on PID was adopted, and recorded as PID-CTM. The actual control torque of each joint of the robot is expressed as follows.

$$
\boldsymbol{u}=\overline{\boldsymbol{M}}(\boldsymbol{\theta})\left(\ddot{\boldsymbol{\theta}}_{d}+\boldsymbol{K}_{P} e+\boldsymbol{K}_{D} \dot{e}+\boldsymbol{K}_{I} \int_{0}^{t} e d_{t}\right)+\overline{\boldsymbol{C}}(\boldsymbol{\theta}, \dot{\boldsymbol{\theta}}) \dot{\boldsymbol{\theta}}+\overline{\boldsymbol{G}}(\boldsymbol{\theta})
$$

where $\boldsymbol{K}_{P}, \boldsymbol{K}_{D}$, and $\boldsymbol{K}_{I}$ are control parameters of the computed torque method. $\boldsymbol{K}_{P}=$ $[5500,6500,5500,6500]^{\mathrm{T}}, \boldsymbol{K}_{I}=[200,200,200,200]^{\mathrm{T}}, \boldsymbol{K}_{D}=[80,80,80,80]^{\mathrm{T}}$.

\subsection{Simulation Results and Discussion}

To compare the performances of the control algorithms, the patient's interference with the lower limb rehabilitation exoskeleton robot was regarded as step interference. At $1.5 \mathrm{~s}$ and $3.5 \mathrm{~s}, \tau_{d 1}=50 \mathrm{~N} \cdot \mathrm{m}$ and $\tau_{d 2}=75 \mathrm{~N} \cdot \mathrm{m}$ were applied to each joint of the robot for a duration of $0.2 \mathrm{~s}$. These interferences also represent strong environmental interference to the lower limb rehabilitation exoskeleton robot.

The trajectory tracking curve and tracking error curve of each joint of the lower limb rehabilitation exoskeleton robot are shown in Figures $5-8$. RHq, RKq, LHq, and LKq are the desired trajectories of the right hip, right knee, left hip, and left knee joints. All three control methods can achieve robot trajectory tracking to a certain extent, and the maximum tracking error occurs during the interference period. The following is a detailed analysis of the control accuracy and anti-interference performances.

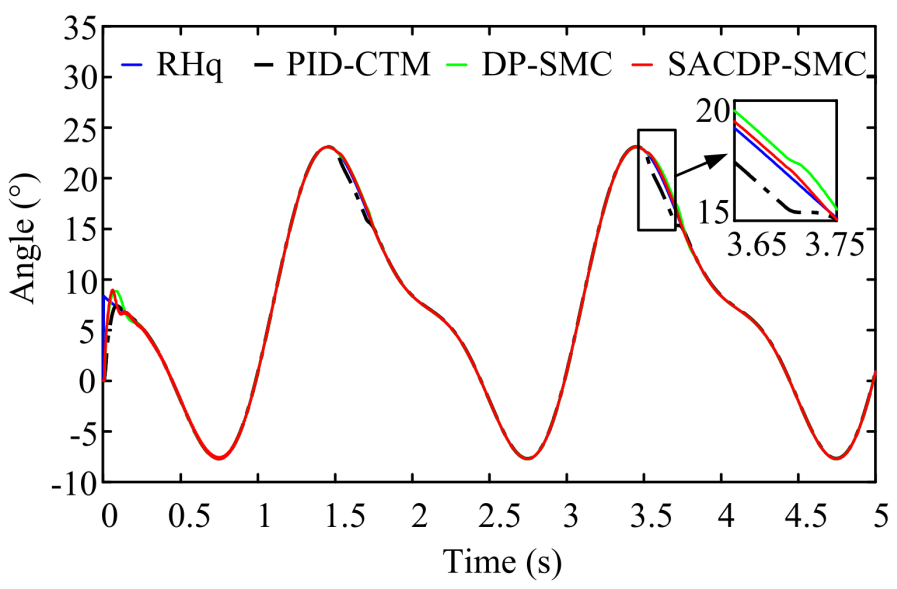

(a)

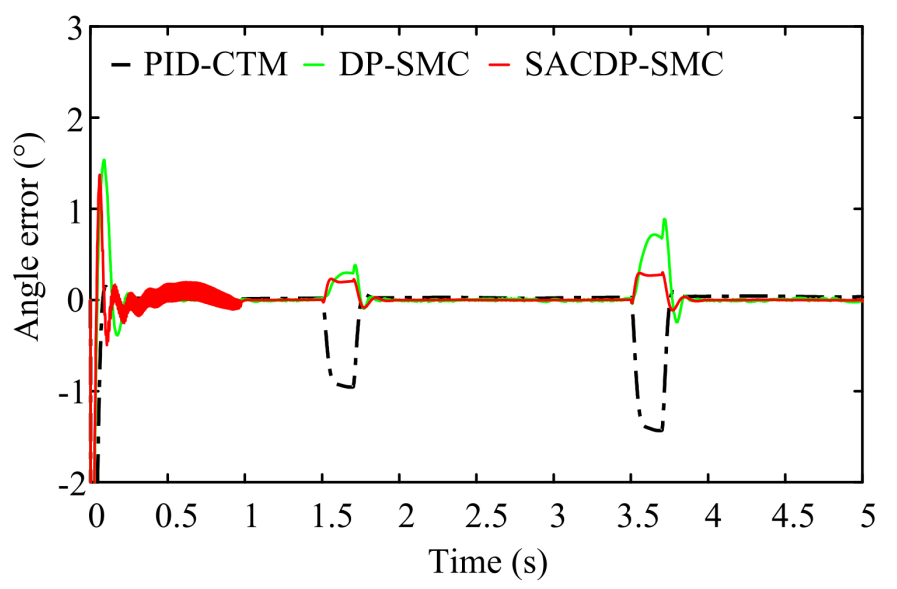

(b)

Figure 5. The tracking curve and error of the right hip. (a) The tracking curve of the right hip; (b) The tracking error of the right hip. 


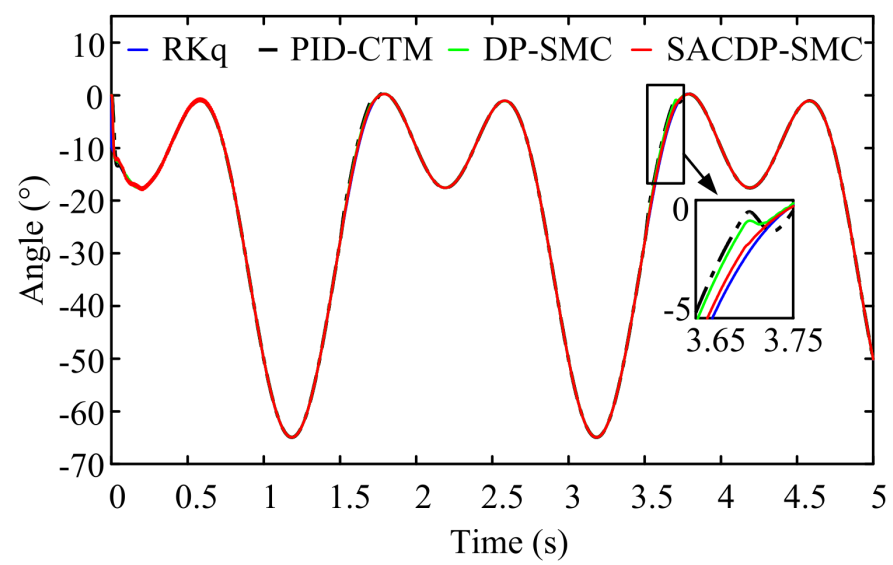

(a)

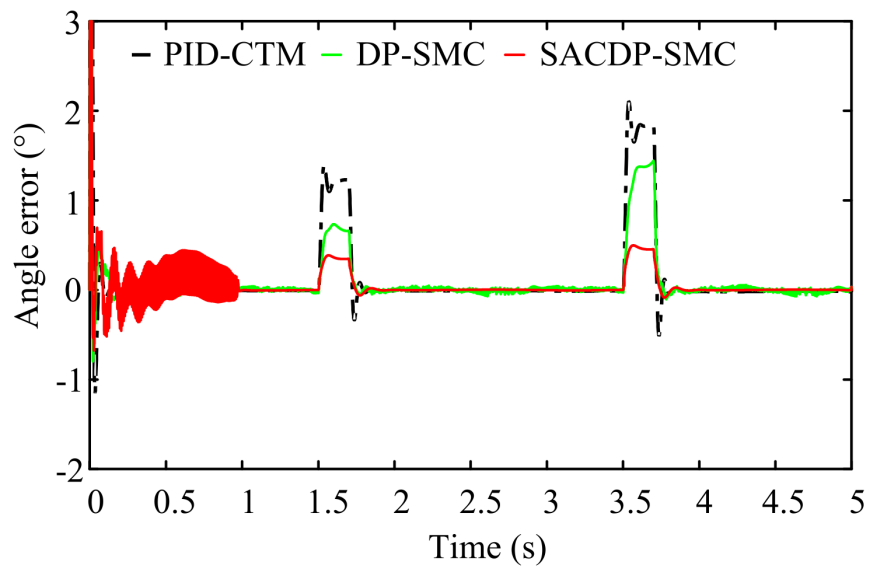

(b)

Figure 6. The tracking curve and error of the right knee. (a) The tracking curve of the right knee; (b) The tracking error of the right knee.

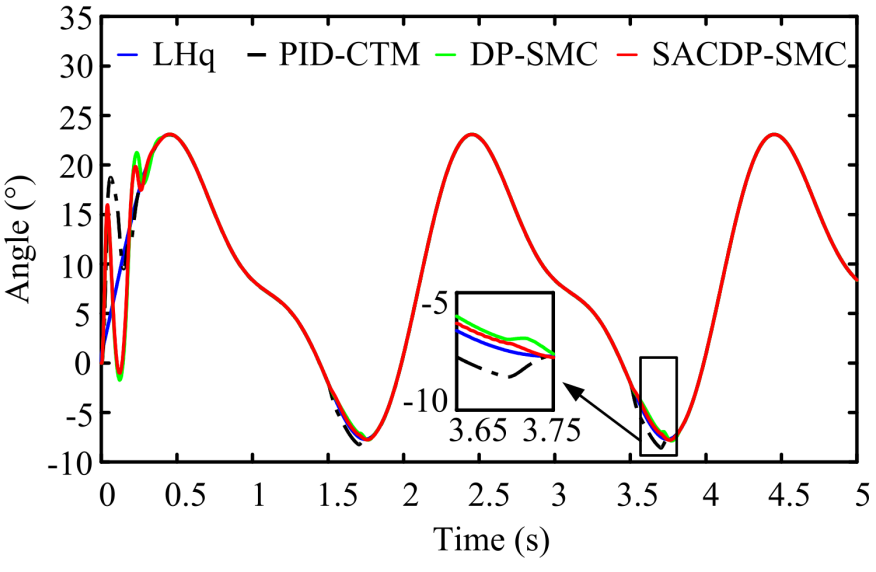

(a)

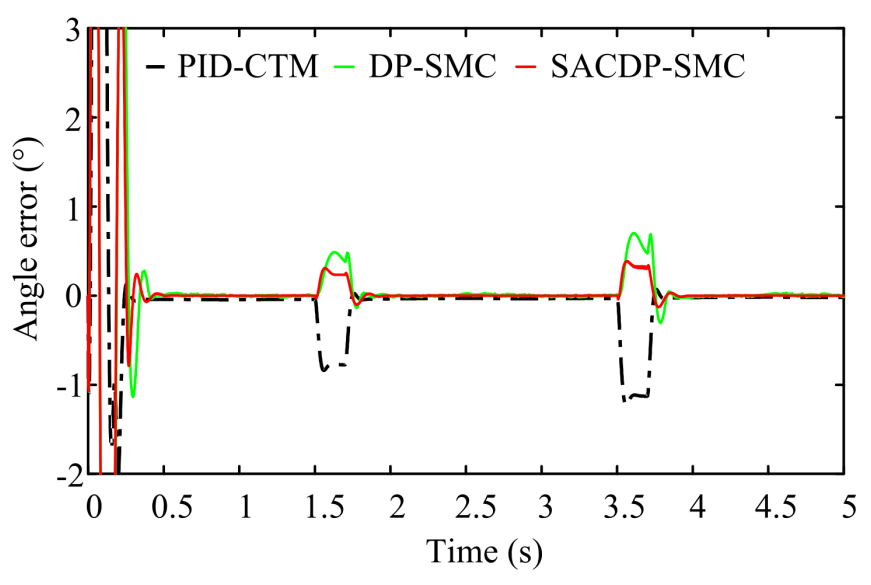

(b)

Figure 7. The angle tracking curve and error of the left hip. (a) The tracking curve of the left hip; (b) The tracking error of the left hip.

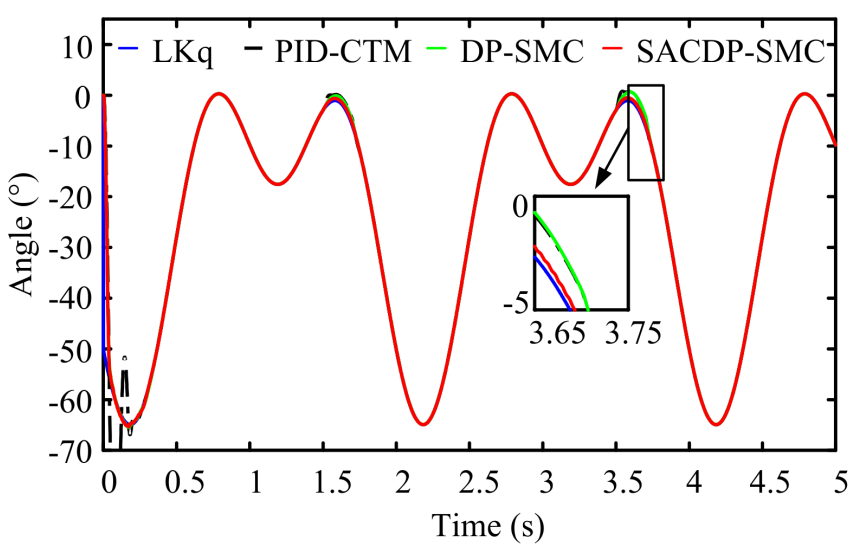

(a)

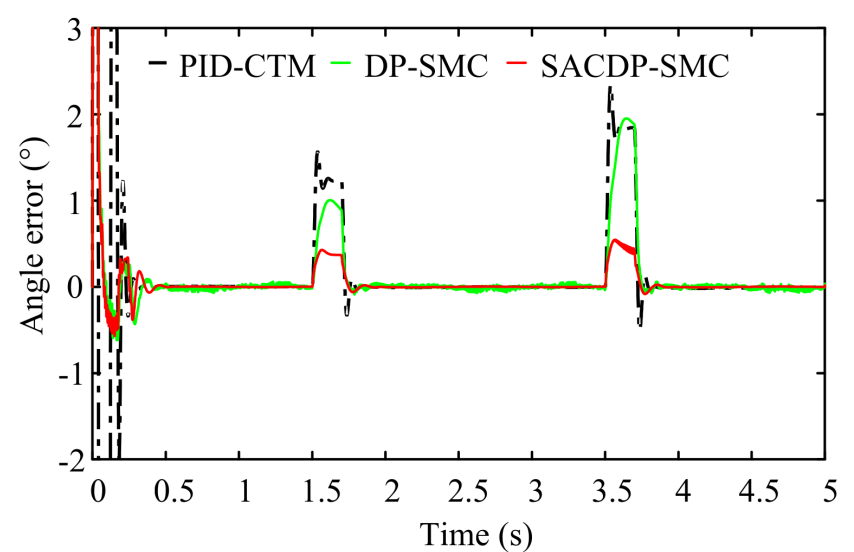

(b)

Figure 8. The angle tracking curve and error of the left knee. (a) The tracking curve of the left knee; (b) The tracking error of the left knee. 
To compare the control accuracy of the three control methods, the maximum absolute tracking error $e_{a}$, the average absolute tracking error $e_{m}$, and the root mean square error $r_{e}$ were used as measurement indicators. When the control system is stable, the tracking error is calculated between $1 \mathrm{~s}$ and $5 \mathrm{~s}$. The maximum absolute tracking error, average tracking absolute error, and root mean square error of the three control methods are shown in Table 4. Compared with the errors when using the traditional computed torque, the percentage of tracking error reduction is shown in Table 4.

Table 4. The metrics of control accuracy.

\begin{tabular}{cccccc}
\hline \multirow{2}{*}{ Indicators } & PID-CTM & \multicolumn{2}{c}{ DP-SMC } & \multicolumn{2}{c}{ SACDP-SMC } \\
\cline { 2 - 6 } & Value $^{\circ}$ & Value $^{\circ}$ & Reduction & Value $^{\circ}$ & Reduction $^{\circ}$ \\
\hline$e_{a}$ & 2.3405 & 1.9533 & $16.54 \%$ & 0.5474 & $76.61 \%$ \\
$e_{m}$ & 0.1679 & 0.1418 & $15.54 \%$ & 0.0474 & $71.77 \%$ \\
$r_{e}$ & 0.5012 & 0.4199 & $16.22 \%$ & 0.1331 & $73.44 \%$ \\
\hline
\end{tabular}

In terms of Table 4, it is clear that the maximum absolute error, average absolute error, and root mean square error of the DP-SMC were reduced by $16.54 \%, 15.54 \%$, and $16.22 \%$, respectively, compared with the PID-CTM under the interference of the patient. In addition, the maximum absolute error of the SACDP-SMC, the mean absolute error, and the root mean square error were reduced by $76.61 \%, 71.77 \%$, and $73.44 \%$, respectively. Both the DP-SMC and SACDP-SMC effectively reduce the trajectory tracking error and improve control accuracy, but the SACDP-SMC achieves the best control accuracy. Even if the patient simultaneously generates $75 \mathrm{Nm}$ of interference torque on each joint of the robot, the maximum absolute error of the proposed SACDP-SMC was only $0.5474^{\circ}$.

Based on the maximum absolute error $e_{a}$; the average absolute error $e_{m}$; and the root and mean square error $r_{e}$; the SACDP-SMC proposed in this paper sharply reduced the trajectory tracking error of the lower limb rehabilitation exoskeleton robot and improved the control accuracy.

To compare the anti-interference capabilities of the three control methods, the error variation $\Delta e$ and the interference adjustment time $\Delta t$ were used as measurement indicators. Under patient interference, the maximum error variations of the three control methods are shown in Table 5.

Table 5. The error variation of the three control methods.

\begin{tabular}{cccccc}
\hline \multirow{2}{*}{$\Delta e$} & PID-CTM & \multicolumn{2}{c}{ DP-SMC } & \multicolumn{2}{c}{ SACDP-SMC } \\
\cline { 2 - 6 } & Value $^{\circ}$ & Value $^{\circ}$ & Reduction & Value $^{\circ}$ & Reduction \\
\hline$\Delta e_{\mathrm{d} 1}$ & 1.5400 & 1.0120 & $34.29 \%$ & 0.4153 & $73.03 \%$ \\
$\Delta e_{\mathrm{d} 2}$ & 2.3350 & 1.9350 & $17.13 \%$ & 0.5425 & $76.77 \%$ \\
\hline
\end{tabular}

In terms of Table 5, compared with PID-CTM, the maximum error variation of DP-SMC was reduced by $34.29 \%$ and $17.13 \%$, and the maximum error variation of SACDP-SMC was reduced by $73.03 \%$ and $76.77 \%$. Both the DP-SMC and SACDP-SMC effectively reduced the impact of interference, but the SACDP-SMC achieved the best anti-interference effect. When the patient simultaneously generated $75 \mathrm{Nm}$ of interference torque on each joint, the maximum error variation of SACDP-SMC was $0.5425^{\circ}$.

Table 6 shows the adjustment times $\Delta t_{d 1}$ and $\Delta t_{d 2}$ of the three control methods for interference $\tau_{d 1}$ and $\tau_{d 2}$. Compared with the PID-CTM, the maximum adjustment time of the DP-SMC was reduced by $16.61 \%$ and $14.46 \%$, and the maximum adjustment time of the SACDP-SMC was reduced by $33.19 \%$ and $30.54 \%$. Both the DP-SMC and SACDP-SMC effectively reduced the interference adjustment time and accelerated the error convergence speed, however, the convergence speed of SACDP-SMC was the best. 
Table 6. The adjusting time of the three control methods.

\begin{tabular}{cccccc}
\hline \multirow{2}{*}{$\Delta t$} & PID-CTM & \multicolumn{2}{c}{ DP-SMC } & \multicolumn{2}{c}{ SACDP-SMC } \\
\cline { 2 - 6 } & Value/s & Value/s & Reduction & Value/s & Reduction \\
\hline$\Delta t_{\mathrm{d} 1}$ & 0.2257 & 0.1882 & $16.61 \%$ & 0.1508 & $33.19 \%$ \\
$\Delta t_{\mathrm{d} 2}$ & 0.2780 & 0.2378 & $14.46 \%$ & 0.1931 & $30.54 \%$ \\
\hline
\end{tabular}

It can be seen from the error variation $\Delta e$ and the interference adjustment time $\Delta t$ that the proposed SACDP-SMC improves the anti-interference ability of the lower limb rehabilitation exoskeleton robot by a large margin.

\subsection{Wearable Experimental Results and Discussion}

To verify the effectiveness of the self-adaptive-coefficient double power sliding mode control method, a wearable experiment was conducted on the lower limb rehabilitation exoskeleton robot prototype using selected healthy testers. The gait trajectory tracked by the lower limb rehabilitation exoskeleton robot still adopted the trajectory curve shown in Equation (21), and during the experiment, the lower limb of the tester was completely driven by the lower limb rehabilitation exoskeleton robot. Figure 9 shows the seven intermediate motion states taken from the video of a complete gait cycle recorded in the experiment. The tracking trajectory curve of each robot joint is shown in Figures 10-13, and the tracking error curve is shown in Figure 14.

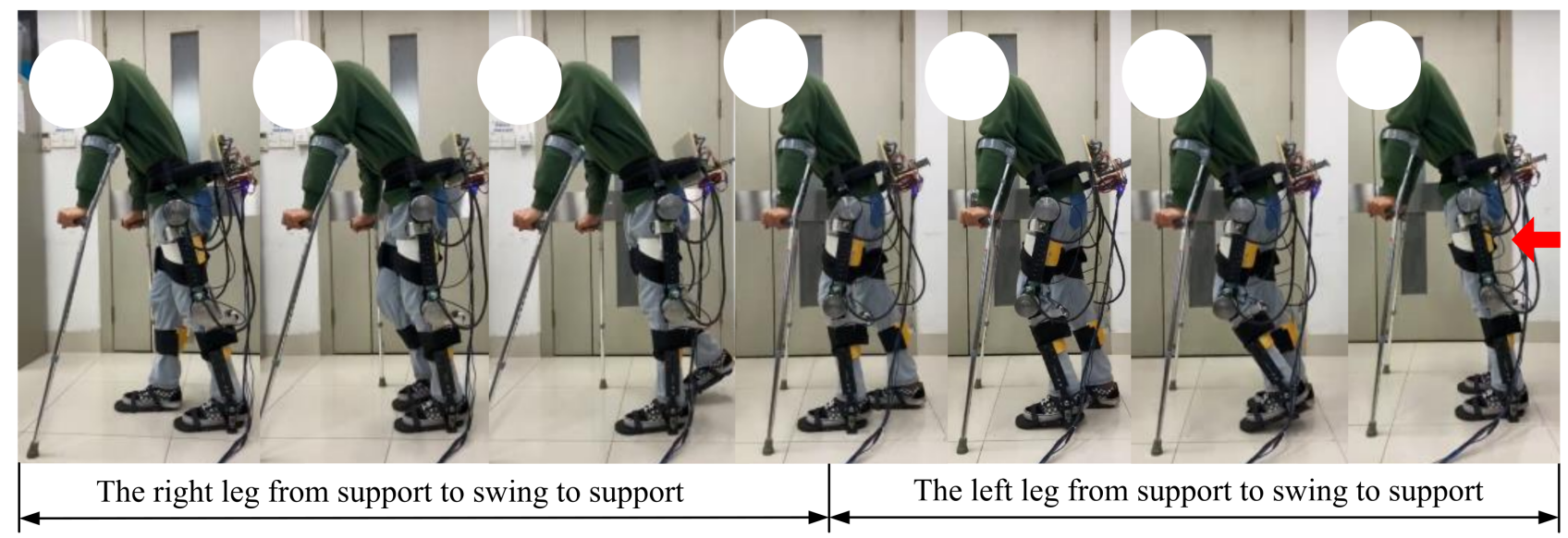

Figure 9. Motion states of the tester in complete gait cycle.

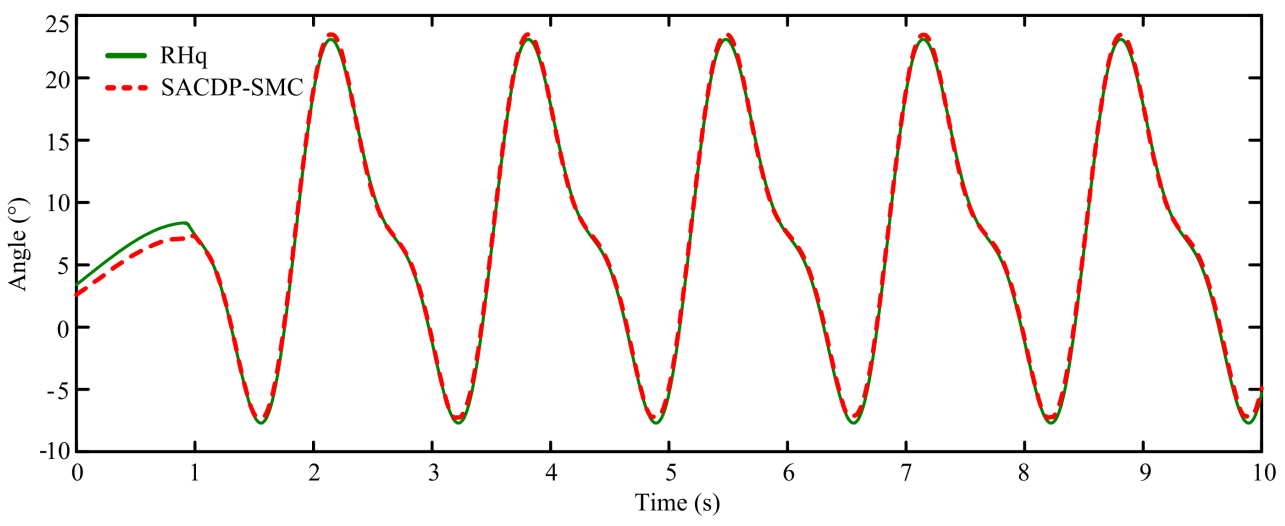

Figure 10. Trajectory tracking curve of the right hip joint. 


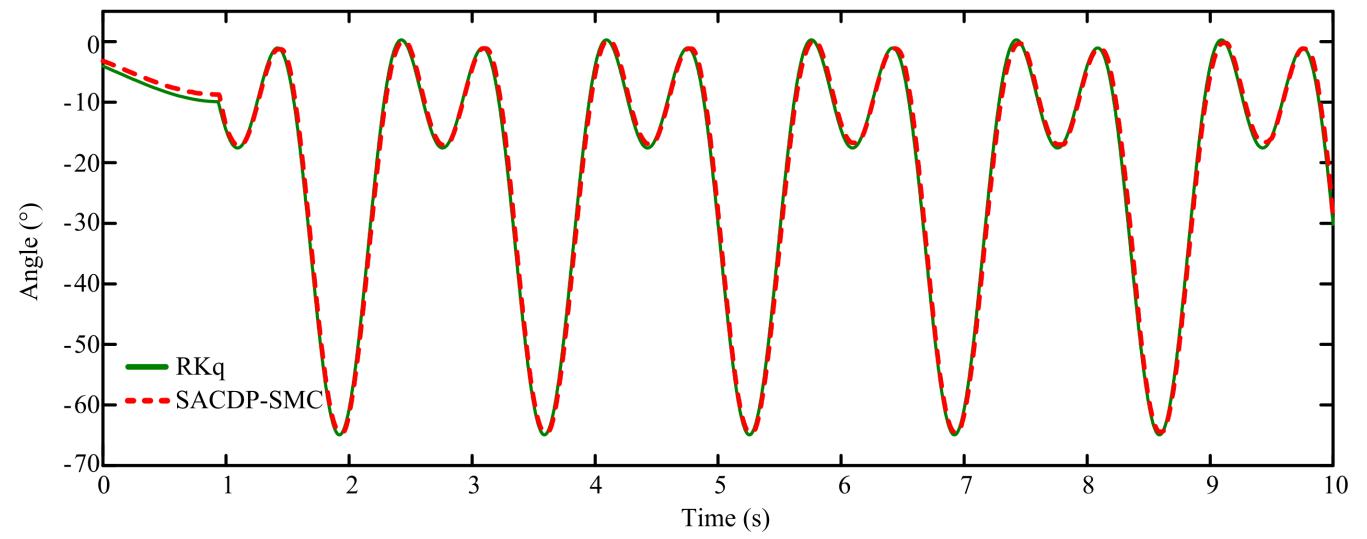

Figure 11. Trajectory tracking curve of the right knee joint.

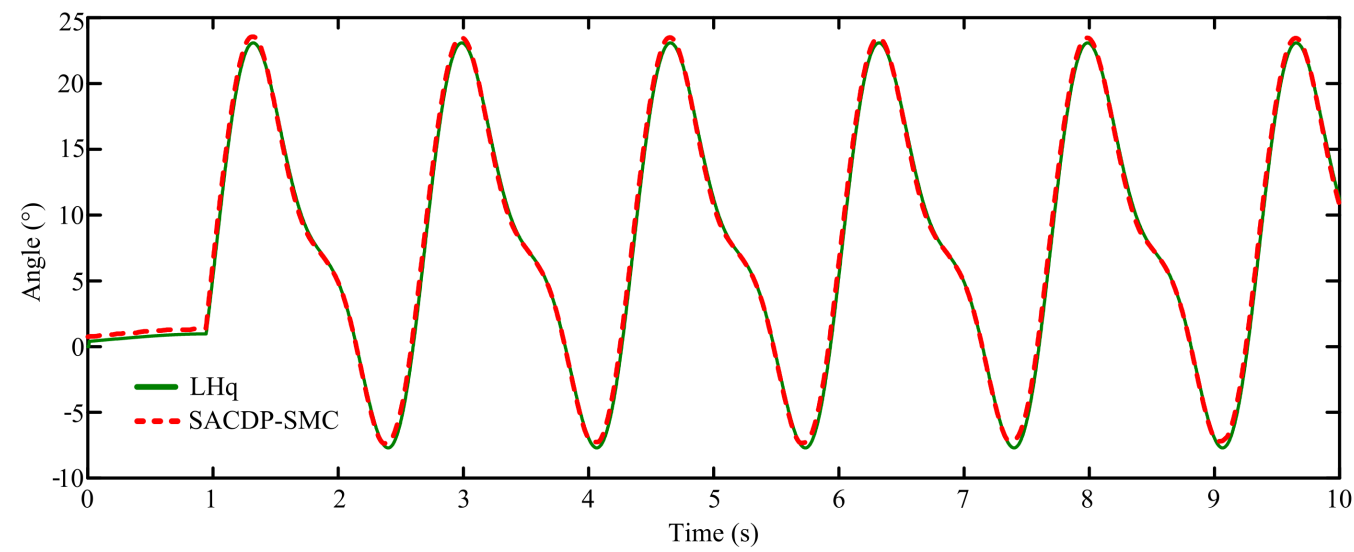

Figure 12. Trajectory tracking curve of the left hip joint.

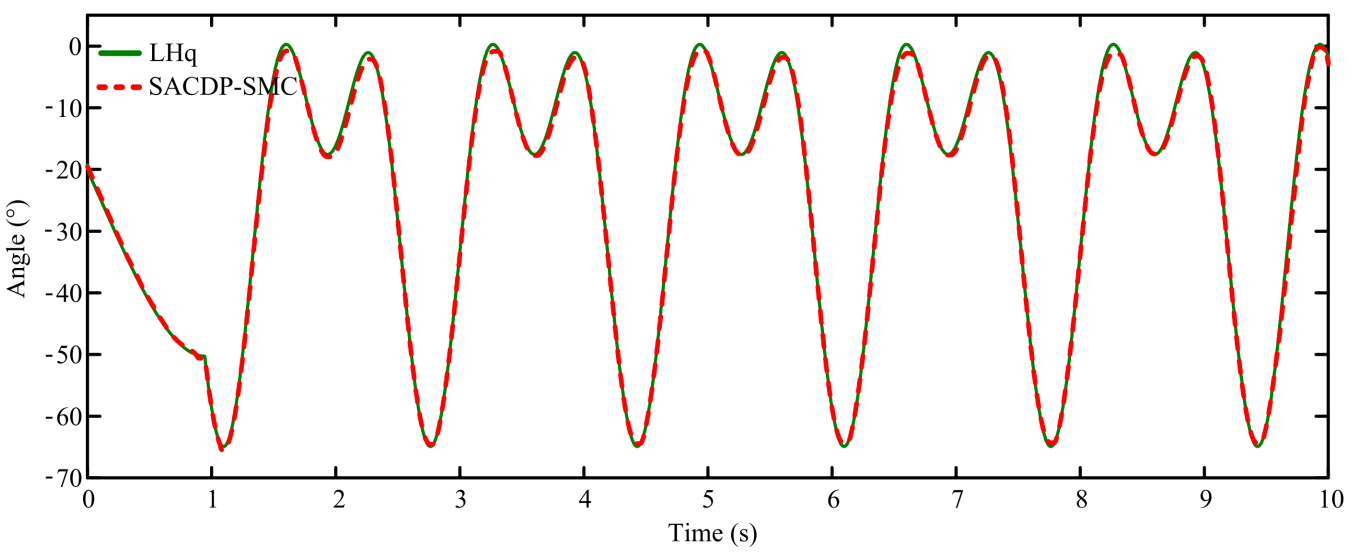

Figure 13. Trajectory tracking curve of the left knee joint. 


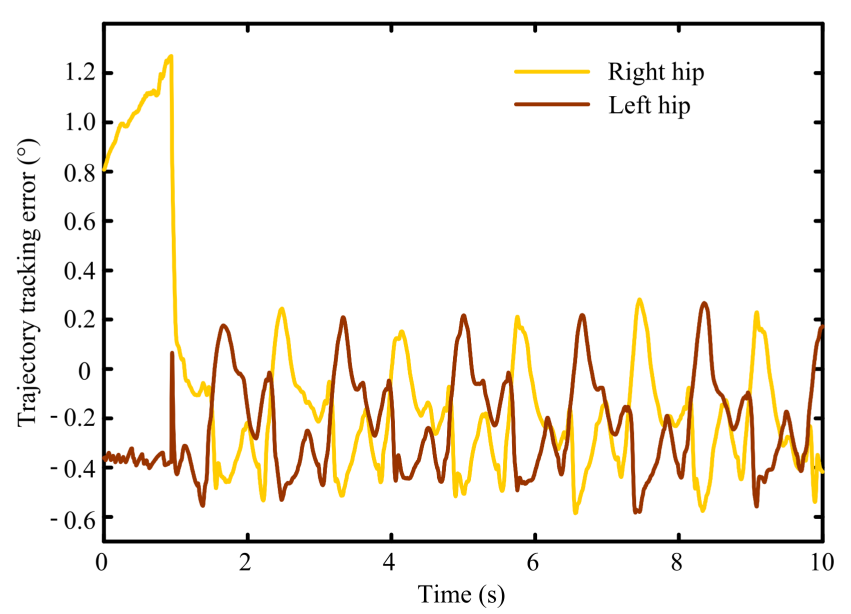

(a)

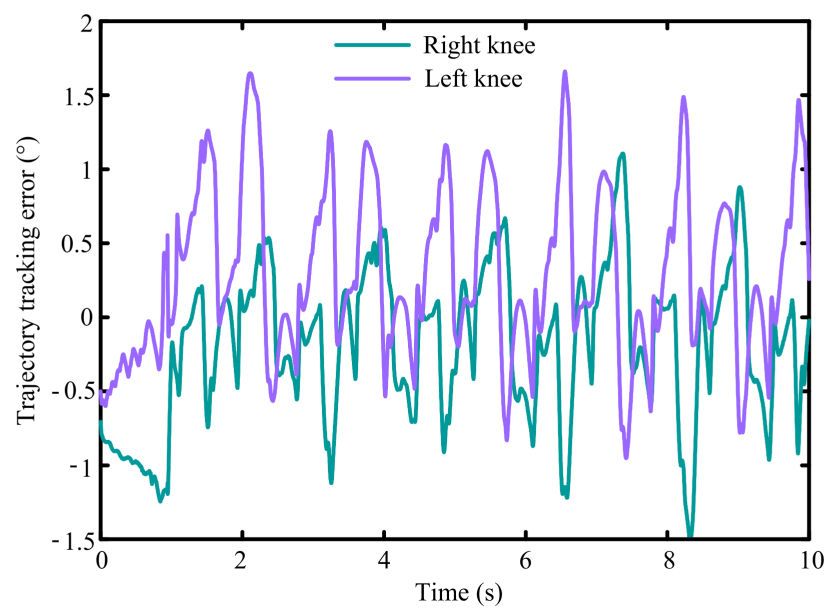

(b)

Figure 14. Trajectory tracking error curve of the (a) hip joint and (b) knee joint.

Figures 9-13 show that based on the self-adaptive-coefficient double power sliding mode control method, the trajectory tracking error of the left and right hip joints ranged from $-0.55^{\circ}$ to $0.2^{\circ}$, and the trajectory tracking error of the left and right knee joints ranged from $-1.5^{\circ}$ to $1.65^{\circ}$ when the lower limb rehabilitation exoskeleton robot prototype drives the tester to track the gait trajectory. Compared with the previous simulation results, the tracking error change of the hip joint was unobvious. The tracking error of the knee joint was increased, but it still had good tracking accuracy. Human gait movement was uncertain. Therefore, the movement of the tester wearing the lower limb rehabilitation exoskeleton robot can be regarded as a random disturbance. When the human wearing the exoskeleton carries out the gait experiment, the tracking error was very small. This shows that the self-adaptive-coefficient double power sliding mode control method proposed in this paper not only achieved good control accuracy, but also had good robustness. In this analysis, the tester was wearing the lower limb rehabilitation exoskeleton robot while walking on the ground, thus, when the legs were grounded, they were greatly disturbed by the ground, which increased the tracking error of the knee joint. In the follow-up research, we will deeply analyze the impact of the ground on the lower limb rehabilitation exoskeleton robot, establish the corresponding mathematical model, and design a controller with better performance.

\section{Conclusions}

In this article, a self-adaptive-coefficient double-power sliding mode control was proposed for trajectory tracking control of the lower limb rehabilitation exoskeleton robot. This method combines the estimated dynamic model of the robot and the sliding mode control based on the double-power reaching law. A nonlinear control law was designed based on the dynamic model of the robot and the method of calculating torque. The compensation term of the control law was introduced and the self-adaptive-coefficient of the compensation term of the control law was designed to reduce the interference of model error and environmental factors and improved the anti-interference ability of the robot.

Simulation analyses of the self-adaptive-coefficient double-power sliding mode control method, the double-power sliding mode control method, and the traditional computed torque method were performed. The results show that the self-adaptive-coefficient doublepower sliding mode control method greatly improved the trajectory tracking accuracy and anti-interference ability.

The simulation showed that the self-adaptive-coefficient double-power sliding mode control method could reduce the trajectory tracking error by more than $71.77 \%$ compared with the traditional computed torque method. Even under strong environmental interferences $(50 \mathrm{Nm}$ and $75 \mathrm{Nm}$ ), the maximum absolute tracking error of the robot remained below $0.5474^{\circ}$. The wearable experiment shows that the lower limb rehabilitation exoskele- 
ton robot had good robustness, and the maximum absolute error of the hip joint and knee joint remained below $0.55^{\circ}$ and $1.65^{\circ}$, respectively.

Author Contributions: Conceptualization, Y.Z. and G.C.; Methodology, Y.Z. and G.C.; Software, Y.Z.; Validation, Y.Z., W.L., and L.L.; Investigation, Y.Z.; Writing—original draft preparation, Y.Z.; Writing - review and editing, Y.Z., G.C., D.D., and J.C.; Visualization, Y.Z., L.L., and W.L.; Supervision, G.C.; Project administration, G.C. All authors have read and agreed to the published version of the manuscript.

Funding: This research was funded by the National Natural Science Foundation of China under Grant NSFC (grant number U1813212) and the Shenzhen Government Fund (grant number JSGG20200701095406010).

Institutional Review Board Statement: The study was conducted according to the guidelines of the Declaration of Helsinki, and approved by the Institutional Review Board (or Ethics Committee) of Shenzhen University (protocol code PN-2021-018 and date of approval 2021-5-8).

Informed Consent Statement: Informed consent was obtained from all subjects involved in the study. Written informed consent has been obtained from the patient(s) to publish this paper.

Data Availability Statement: Not applicable.

Conflicts of Interest: The authors declare no conflict of interest.

\section{References}

1. Vaida, C.; Carbone, G.; Major, K.; Major, Z.; Plitea, N.; Pisla, D. On human robot interaction modalities in the upper limb rehabilitation after stroke. Acta Tech. Napoc. Ser. Math. Mech. Eng. 2017, 60, 91-102.

2. Tucan, P.; Vaida, C.; Plitea, N.; Pisla, A.; Carbone, G.; Pisla, D. Risk-based assessment engineering of a Parallel Robot used in post-stroke upper limb rehabilitation. Sustainability 2019, 11, 2893. [CrossRef]

3. Benson, I.; Hart, K.; Tussler, D.; Van Middendorp, J.J. Lower-limb exoskeletons for individuals with chronic spinal cord injury: Findings from a feasibility study. Clin. Rehabil. 2016, 30, 73-84. [CrossRef] [PubMed]

4. Li, N.; Yang, T.; Yu, P.; Chang, J.; Zhao, L.; Zhao, X.; Elhajj, I.H.; Xi, N.; Liu, L. Bio-inspired upper limb soft exoskeleton to reduce stroke-induced complications. Bioinspir. Biomim. 2018, 13, 1-25. [CrossRef]

5. Hobbs, B.; Artemiadis, P. A review of robot-assisted lower-limb stroke therapy: Unexplored paths and future directions in gait rehabilitation. Front. Neurorobot. 2020, 14, 1-16. [CrossRef]

6. Pisla, D.; Nadas, I.; Tucan, P.; Albert, S.; Carbone, G.; Antal, T.; Banica, A.; Gherman, B. Development of a control system and functional validation of a parallel robot for lower limb rehabilitation. Actuators 2021, 10, 277. [CrossRef]

7. Vaida, C.; Birlescu, I.; Pisla, A.; Ulinici, I.M.; Tarnita, D.; Carbone, G.; Pisla, D. Systematic design of a parallel robotic system for lower limb rehabilitation. IEEE Access 2020, 8, 34522-34537. [CrossRef]

8. Bernhardt, M.; Frey, M.; Colombo, G.; Riener, R. Hybrid force-position control yields cooperative behaviour of the rehabilitation robot LOKOMAT. In Proceedings of the 9th International Conference on Rehabilitation Robotics, Chicago, IL, USA, 28 June-1 July 2005; pp. 536-539.

9. Kawabata, T.; Satoh, H.; Sankai, Y. Working posture control of robot suit HAL for reducing structural stress. In Proceedings of the 2009 IEEE International Conference on Robotics and Biomimetics (ROBIO), Guilin, China, 19-23 December 2009; pp. 2013-2018.

10. Talaty, M.; Esquenazi, A.; Briceno, J.E. Differentiating Ability in users of the ReWalkTM Powered Exoskeleton: An analysis of walking kinematics. In Proceedings of the 2013 IEEE 13th International Conference on Rehabilitation Robotics (ICORR), Seattle, WA, USA, 24-26 June 2013; pp. 1-5.

11. Zhou, S.H.; Tan, Y.; Oetomo, D.; Freeman, C.; Burdet, E.; Mareels, I. Modeling of endpoint feedback learning implemented through point-to-point learning control. IEEE Trans. Control Syst. Technol. 2017, 25, 1576-1585. [CrossRef]

12. Campbell, S.M.; DIduch, C.P.; Sensinger, J.W. Autonomous Assistance-as-needed control of a lower limb exoskeleton with guaranteed stability. IEEE Access 2020, 8, 51168-51178. [CrossRef]

13. Chen, C.; Zhang, S.; Zhu, X.; Shen, J.; Xu, Z. Disturbance observer-based patient-cooperative control of a lower extremity rehabilitation exoskeleton. Int. J. Precis. Eng. Manuf. 2020, 21, 957-968. [CrossRef]

14. Amiri, M.S.; Ramli, R.; Ibrahim, M.F. Hybrid design of PID controller for four DoF lower limb exoskeleton. Appl. Math. Model. 2019, 72, 17-27. [CrossRef]

15. Han, S.; Wang, H.; Tian, Y. Adaptive computed torque control based on RBF network for a lower limb exoskeleton. In Proceedings of the 2018 IEEE 15th International Workshop on Advanced Motion Control (AMC), Tokyo, Japan, 9-11 March 2018; pp. 35-40.

16. Sun, W.; Lin, J.-W.; Su, S.-F.; Wang, N.; Er, M.J. Reduced Adaptive fuzzy decoupling control for lower limb exoskeleton. IEEE Trans. Cybern. 2020, 51, 1099-1109. [CrossRef]

17. Han, S.; Wang, H.; Tian, Y.; Christov, N. Time-delay estimation based computed torque control with robust adaptive RBF neural network compensator for a rehabilitation exoskeleton. ISA Trans. 2020, 97, 171-181. [CrossRef] 
18. Ahmed, S.F.; Raza, Y.; Mahdi, H.F.; Wan Muhamad, W.M.; Kamran Joyo, M.; Shah, A.; Koondhar, M.Y. Review on sliding mode controller and its modified types for rehabilitation robots. In Proceedings of the 2019 IEEE 6th International Conference on Engineering Technologies and Applied Sciences (ICETAS), Kuala Lumpur, Malaysia, 20-21 December 2019.

19. Brahmi, B.; Bojairami, I.E.; Saad, M.; Driscoll, M.; Zemam, S.; Laraki, M.H. Enhancement of sliding mode control performance for perturbed and unperturbed nonlinear systems: Theory and experimentation on rehabilitation robot. J. Electr. Eng. Technol. 2021, 16, 599-616. [CrossRef]

20. Liu, J.; Zhang, Y.; Wang, J.; Chen, W. Adaptive sliding mode control for a lower-limb exoskeleton rehabilitation robot. In Proceedings of the 2018 13th IEEE Conference on Industrial Electronics and Applications (ICIEA), Wuhan, China, 31 May-2 June 2018; pp. 1481-1486.

21. Du, F.; Sun, Y.; Li, G.; Jiang, G.; Kong, J.; Jiang, D.; Li, Z. Adaptive fuzzy sliding mode control algorithm simulation for 2-DOF articulated robot. Int. J. Wirel. Mob. Comput. 2017, 13, 306-313. [CrossRef]

22. Teng, L.; Bai, S. Fuzzy sliding mode control of an upper-limb exoskeleton robot. In Proceedings of the 2019 IEEE International Conference on Cybernetics and Intelligent Systems (CIS) and IEEE Conference on Robotics, Automation and Mechatronics (RAM), Bangkok, Thailand, 18-20 November 2019; pp. 12-17.

23. Islam, M.R.; Rahmani, M.; Rahman, M.H. A novel exoskeleton with fractional sliding mode control for upper limb rehabilitation. Robotica 2020, 38, 2099-2120. [CrossRef]

24. Nair, A.S.; Ezhilarasi, D. Performance Analysis of super twisting sliding mode controller by ADAMS-MATLAB Co-simulation in lower extremity exoskeleton. Int. J. Precis. Eng. Manuf. Green Technol. 2020, 7, 743-754. [CrossRef]

25. Hao, Z.; Liu, K.; Wei, Q. Adaptive Neural Network Control of Lower Limb Exoskeleton Robots Using Disturbance Observer. In Proceedings of the 2020 5th International Conference on Advanced Robotics and Mechatronics (ICARM), Shenzhen, China, 18-21 December 2020; pp. 246-251.

26. Long, Y.; Peng, Y. Extended State observer-based nonlinear terminal sliding mode control with feedforward compensation for lower extremity exoskeleton. IEEE Access 2021, 1. [CrossRef]

27. Chen, C.F.; Du, Z.J.; He, L.; Wang, J.Q.; Wu, D.M.; Dong, W. Active disturbance rejection with fast terminal sliding mode control for a lower limb exoskeleton in swing phase. IEEE Access 2019, 7, 72343-72357. [CrossRef]

28. Devika, K.B.; Thomas, S. Power rate exponential reaching law for enhanced performance of sliding mode control. Int. J. Control. Autom. Syst. 2017, 15, 2636-2645.

29. Xia, Y.; Xie, W.; Ma, J. Research on trajectory tracking control of manipulator based on modified terminal sliding mode with double power reaching law. Int. J. Adv. Robot. Syst. 2019, 16, 1-10. [CrossRef]

30. Kang, Z.; Yu, H.; Li, C. Variable-parameter double-power reaching law sliding mode control method*. Automatika 2020, 61, 345-351. [CrossRef]

31. Hernández, J.H.; Cruz, S.S.; López-Gutiérrez, R.; González-Mendoza, A.; Lozano, R. Robust nonsingular fast terminal slidingmode control for Sit-to-Stand task using a mobile lower limb exoskeleton. Control Eng. Pract. 2020, 101, 104496. [CrossRef]

32. Van, M.; Do, X.P.; Mavrovouniotis, M. Self-tuning fuzzy PID-nonsingular fast terminal sliding mode control for robust fault tolerant control of robot manipulators. ISA Trans. 2020, 96, 60-68. [CrossRef] [PubMed]

33. Ren, T.; Dong, Y.; Wu, D.; Chen, K. Collision detection and identification for robot manipulators based on extended state observer. Control Eng. Pract. 2018, 79, 144-153. [CrossRef]

34. Reppa, V.; Tzes, A. Fault detection based on orthotopic set membership identification for robot manipulators. IFAC Proc. Vol. 2008, 17, 7344-7349. [CrossRef]

35. Xie, Y.; Zhang, X.; Meng, W.; Zheng, S.; Jiang, L.; Meng, J.; Wang, S. Coupled fractional-order sliding mode control and obstacle avoidance of a four-wheeled steerable mobile robot. ISA Trans. 2021, 108, 282-294. [CrossRef]

36. La Salle, J.P. The Stability of Dynamical Systems; SIAM: Philadelphia, PA, USA, 1976; ISBN 0898710227. 